\title{
Transient 3d contact problems-NTS method: mixed methods and conserving integration
}

\author{
Christian Hesch • Peter Betsch
}

Received: 1 October 2010 / Accepted: 28 March 2011 / Published online: 12 April 2011

(C) Springer-Verlag 2011

\begin{abstract}
The present work deals with a new formulation for transient large deformation contact problems. It is well known, that one-step implicit time integration schemes for highly non-linear systems fail to conserve the total energy of the system. To deal with this drawback, a mixed method is newly proposed in conjunction with the concept of a discrete gradient. In particular, we reformulate the well known and widely-used node-to-segment methods and establish an energy-momentum scheme. The advocated approach ensures robustness and enhanced numerical stability, demonstrated in several three-dimensional applications of the proposed algorithm.
\end{abstract}

Keywords Contact · Energy methods - Large deformation

\section{Introduction}

The objective of this paper is to develop a new time integration scheme with enhanced numerical stability properties for unilateral contact within the domain of large deformation problems. The concept of the underlying nodeto-segment (NTS) approach, originally introduced by Hallquist [14], has been subject of various papers published within the last 30 years. A survey of previous developments can be found in the textbooks written by Laursen [24] and Wriggers [31]. In contrast to that, the far more complex mortar constraints (cf. [19,27,30] and the references therein) are currently subject of intensive research. We will deal with this kind of constraints in a subsequent paper. The main goal of the present paper is to develop adequate time integration

C. Hesch $(\varangle) \cdot$ P. Betsch

Chair of Computational Mechanics, Department of Mechanical

Engineering, University of Siegen, Siegen, Germany

e-mail: hesch@imr.mb.uni-siegen.de schemes for unilateral contact constraints in the context of the NTS method.

Conserving time integration schemes for non-linear elastodynamics are widely used. Their development started in the early 1990s of the past century with the work of Simo et al. [28,29] and has been extended to the general concept of a discrete gradient by Gonzalez [10,12]. Energy-decaying variants of conserving integrators have been developed by Armero and Romero [2,3]. We refer to Betsch and Steinmann [6-8] for further details about conserving integrators for mechanical systems.

Energy conserving time integration schemes for unilateral contact problems have been developed previously within the framework of the NTS method (e.g. [1,13,25,26]). The extension of the notion of a discrete gradient to contact problems has been developed by Hauret and Le Tallec [15] and Betsch and Hesch [5]. Note, however, that the former approach fails to conserve angular momentum, while the latter treatment is confined to two-dimensional problems.

Mixed or reducible formulations are well-established in present-day finite element methods, see Zienkiewicz et al. $[35,36]$. A general mixed approach relies on the introduction of additional fields by employing the $\mathrm{Hu}$-Washizu functional. We extend this concept to reformulate the NTS constraints. The specific method used in this paper can be regarded as a coordinate augmentation technique, originally introduced by Betsch and Uhlar [9] in the context of multibody dynamics for the description of joint-coordinates and conjugate jointforces. An application of this approach to domain decomposition problems can be found in Hesch and Betsch [17].

An outline of the present work is as follows. Section 2 gives a short introduction to the governing equations relevant for large deformation contact problems. In Sect. 3 a finite element discretization of the continuous problem under consideration is developed. Section 4 provides the mixed 
formulation of the specific contact constraints, which facilitates the design of an energy-momentum scheme. The semidiscrete equations of motion are derived in Sect. 5, together with the discretization in time and the newly developed energy-momentum scheme. Representative numerical examples are presented in Sect. 6. Eventually, conclusions are drawn in Sect. 7.

\section{Governing equations}

For the large deformation problem under consideration we define the sets $\Omega^{(i)} \subset \mathbb{R}^{3}, i \in\{1, \ldots, k\}$ as indicated in Fig. 1, representing the reference configuration of the involved bodies. For convenience we restrict ourselves to a two body contact problem $i \in\{1,2\}$ excluding self contact (see $[4,34]$ ) without prejudice to the generality of the further development. The potential areas of contact are defined on the surfaces $\partial \Omega^{(i)}=\Gamma^{(i)}$, which are subdivided into the Dirichlet boundary $\Gamma_{u}^{(i)}$ with prescribed displacements, the Neumann boundary $\Gamma_{\sigma}^{(i)}$ with prescribed tractions and $\Gamma_{c}^{(i)}$, the potential contact area. It is required that the different boundaries satisfy

$\Gamma_{u}^{(i)} \cup \Gamma_{\sigma}^{(i)} \cup \Gamma_{c}^{(i)}=\Gamma^{(i)}$ and

$\Gamma_{u}^{(i)} \cap \Gamma_{\sigma}^{(i)}=\Gamma_{\sigma}^{(i)} \cap \Gamma_{c}^{(i)}=\Gamma_{u}^{(i)} \cap \Gamma_{c}^{(i)}=\emptyset$.

Adopting a Lagrangian framework, we assume a mapping $\varphi^{(i)}\left(X^{(i)}, t\right)$, characterizing the current position at time $t \in$ $\mathbb{I}=[0, T]$ of a material point $\boldsymbol{X}^{(i)} \in \Omega^{(i)}$. The corresponding mapping of the surface $\Gamma^{(i)}$ is denoted by $\gamma^{(i)}=\varphi\left(\Gamma^{(i)}, t\right)$. The prescribed tractions $\overline{\boldsymbol{T}}^{(i)}$ and displacements $\overline{\boldsymbol{\varphi}}^{(i)}$ are stated as follows:

$\boldsymbol{P}^{(i)} \boldsymbol{N}^{(i)}=\overline{\boldsymbol{T}} \quad$ on $\Gamma_{\sigma}^{(i)}$ and $\boldsymbol{\varphi}^{(i)}=\overline{\boldsymbol{\varphi}}^{(i)}$ on $\Gamma_{u}^{(i)}$,

where $\boldsymbol{N}^{(i)}$ denotes the outward unit normal vector in the reference configuration of body $i$ and $\boldsymbol{P}^{(i)}$ the first PiolaKirchhoff stress tensor. In the sequel we make use of the notation

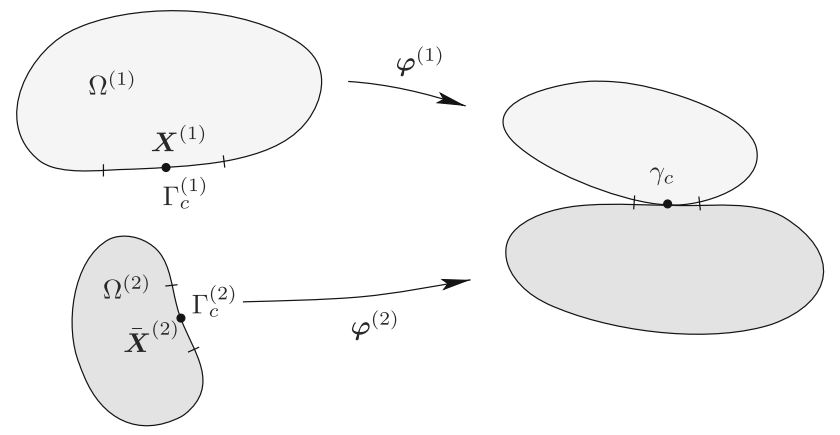

Fig. 1 The two body large deformation contact problem
$\int_{\Omega^{(i)}}(\bullet) \cdot(\bullet) \mathrm{d} \Omega^{(i)}=:\langle\bullet, \bullet\rangle^{(i)}$ and $\int_{\Gamma^{(i)}}(\bullet) \cdot(\bullet) \mathrm{d} \Gamma^{(i)}=:\langle\bullet, \bullet\rangle_{\Gamma}^{(i)}$

The contribution of body $(i)$ to the virtual work for a large deformation contact problem can be expressed as follows

$$
\begin{aligned}
G^{(i)}(\boldsymbol{\varphi}, \delta \boldsymbol{\varphi})= & \left\langle\rho_{R} \ddot{\boldsymbol{\varphi}}, \delta \boldsymbol{\varphi}\right\rangle^{(i)}+\left\langle\boldsymbol{P}, \nabla_{\boldsymbol{X}}(\delta \boldsymbol{\varphi})\right\rangle^{(i)} \\
& -\left\langle\rho_{R} \overline{\boldsymbol{B}}, \delta \boldsymbol{\varphi}\right\rangle^{(i)}-\langle\overline{\boldsymbol{T}}, \delta \boldsymbol{\varphi}\rangle_{\Gamma_{\sigma}}^{(i)}-\langle\boldsymbol{t}, \delta \boldsymbol{\varphi}\rangle_{\Gamma_{c}}^{(i)}
\end{aligned}
$$

The first term on the right-hand side specifies the contribution of the inertia forces where a superposed dot denotes differentiation with respect to time and $\rho_{R}$ the reference mass density. The second term specifies the virtual work of the internal forces using the nabla operator $\nabla_{\boldsymbol{X}}(\delta \boldsymbol{\varphi})=\partial(\delta \boldsymbol{\varphi}) / \partial \boldsymbol{X}$. The third and fourth term represent the virtual work of the external forces. The former takes the body forces into account, where $\overline{\boldsymbol{B}}$ denotes the forces per unit volume, while the latter represents the forces acting on the Neumann boundary, where $\bar{T}$ denotes the forces per unit area. The last term specifies the virtual work associated with the contact tractions $t$. We postulate that the weak form of the balance of linear momentum, given by

$\sum_{i=1}^{2} G^{(i)}(\varphi, \delta \varphi)=0$

must hold for all $t \in \mathbb{I}$. Considering the balance of linear momentum across the current configuration of the contact area

$G^{c}(\boldsymbol{\varphi}, \delta \boldsymbol{\varphi})=-\langle\boldsymbol{t}, \delta \boldsymbol{\varphi}\rangle_{\gamma_{c}}^{(1)}-\langle\boldsymbol{t}, \delta \boldsymbol{\varphi}\rangle_{\gamma_{c}}^{(2)}$

and postulating that the last equation equals zero for each differential element $\mathrm{d} \gamma_{c}$, i.e. $\boldsymbol{t}^{(1)} \mathrm{d} \gamma_{c}^{(1)}=-\boldsymbol{t}^{(2)} \mathrm{d} \gamma_{c}^{(2)}$, the total contribution to the virtual work reads

$G^{c}(\boldsymbol{\varphi}, \delta \boldsymbol{\varphi})=-\left\langle\boldsymbol{t},\left(\delta \boldsymbol{\varphi}^{(1)}-\delta \boldsymbol{\varphi}^{(2)}\right)\right\rangle_{\gamma_{c}}^{(1)}$.

In the case of frictionless contact only the normal component of $\boldsymbol{t}^{(1)}$, defined by $\boldsymbol{t}^{(1)} \cdot \boldsymbol{n}^{(1)}=: \lambda$ is nonzero, where $\boldsymbol{n}^{(1)}$ denotes the outward unit normal vector to $\mathrm{d} \gamma_{c}^{(1)}$. Accordingly, (7) yields

$G^{c}(\boldsymbol{\varphi}, \delta \boldsymbol{\varphi})=-\left\langle\lambda \boldsymbol{n},\left(\delta \boldsymbol{\varphi}^{(1)}-\delta \boldsymbol{\varphi}^{(2)}\right)\right\rangle_{\gamma_{c}}^{(1)}$.

\section{Spatial discretization}

To achieve a numerical solution for the nonlinear problem under consideration, we apply a spatial discretization process to each body $\Omega^{(i)}$ by introducing a set of elements $e \in \mathbb{E}^{\text {h }}$ via

$\Omega^{(i), \mathrm{h}}=\bigcup_{\forall e \in \mathbb{E}^{\mathrm{h}}} \Omega_{e}^{(i), \mathrm{h}}$. 
Using a standard displacement-based finite element approach, we introduce finite dimensional approximations of $\varphi$ and $\delta \varphi$ given by

$\boldsymbol{\varphi}^{(i), \mathrm{h}}=\sum_{A \in \omega} N^{A} \boldsymbol{q}_{A}^{(i)}, \quad$ and $\delta \boldsymbol{\varphi}^{(i), \mathrm{h}}=\sum_{B \in \omega} N^{B} \delta \boldsymbol{q}_{B}^{(i)}$,

where $\boldsymbol{q}_{A}^{(i)}=\varphi^{(i)}\left(X_{A}^{(i)}, t\right), A, B \in \omega=\left\{1, \ldots, n_{\text {node }}\right\}$ are the nodal values of the configuration mapping at time $t \in \mathbb{I}$. Furthermore, $N^{A}\left(\boldsymbol{X}^{(i)}\right): \Omega^{(i), \mathrm{h}} \rightarrow \mathbb{R}$ are the global shape functions associated with nodes $A$. In addition, $N^{C}\left(\boldsymbol{X}^{(i)}\right)$ : $\Gamma^{(1), \mathrm{h}} \rightarrow \mathbb{R}$ are the global shape functions associated with nodes $C \in \bar{\omega}=\left\{1, \ldots, n_{\text {surf }}\right\}$ on the corresponding contact surface $\Gamma^{(1), \mathrm{h}}$.

Note, that we will make use of the summation convention if convenient and unique. Substitution of the above approximations into (5) yields the semi-discrete form of the virtual work principle

$$
\begin{aligned}
G^{\mathrm{h}}(\boldsymbol{q}, \delta \boldsymbol{q})= & \sum_{i=1}^{2} \delta \boldsymbol{q}_{A}^{(i)} \cdot\left[M^{A B} \ddot{\boldsymbol{q}}_{B}^{(i)}+\boldsymbol{f}^{(i), \text { int }, A}\right. \\
& \left.+\boldsymbol{f}^{(i), \mathrm{ext}, A}+\boldsymbol{f}^{(i), \mathrm{c}, A}\right]
\end{aligned}
$$

where the nodal mass contribution is given by

$M^{A B}=\left\langle\rho_{R} N^{A}, N^{B}\right\rangle$,

and the internal nodal forces assume the form

$\boldsymbol{f}^{(i), \text { int }, A}=\left\langle\nabla N^{A}\left(\boldsymbol{X}^{(i)}\right) \cdot \boldsymbol{S}, \nabla N^{B}\left(\boldsymbol{X}^{(i)}\right)\right\rangle \boldsymbol{q}_{B}^{(i)}$.

Here, $\boldsymbol{S}$ denotes the second Piola-Kirchhoff stress tensor. Similarly, the external forces can be written as

$\boldsymbol{f}^{\mathrm{ext}, A}=-\left\langle N^{A}, \rho_{R} \overline{\boldsymbol{B}}\right\rangle^{(i)}-\left\langle N^{A}, \overline{\boldsymbol{T}}\right\rangle_{\Gamma_{\sigma}}^{(i)}$.

Throughout this paper we assume that the internal and the external forces can be associated with a potential energy function of the form

$V(\boldsymbol{q})=\sum_{i=1}^{2}\left(V^{(i), \text { int }}\left(\boldsymbol{q}^{(i)}\right)+V^{(i), \operatorname{ext}}\left(\boldsymbol{q}^{(i)}\right)\right)$

with

$$
\begin{aligned}
& V^{(i), \text { int }}\left(\boldsymbol{q}^{(i)}\right)=\int_{\Omega^{(i)}} W(\boldsymbol{C}) \mathrm{d} V \text { and } \\
& V^{(i), \operatorname{ext}}\left(\boldsymbol{q}^{(i)}\right)=-\boldsymbol{q}_{A} \cdot\left\langle N^{A}, \rho_{R} \overline{\boldsymbol{B}}\right\rangle^{(i)}-\boldsymbol{q}_{A} \cdot\left\langle N^{A}, \overline{\boldsymbol{T}}\right\rangle_{\Gamma_{\sigma}}^{(i)}
\end{aligned}
$$

using the right Cauchy-Green tensor $\boldsymbol{C}$ and a strain energy density function $W(\boldsymbol{C})$. For further details we refer to standard finite element textbooks (e.g. [20]).

At last, we have to discretize the virtual work associated with the contact interface. Therefore, we introduce below the node-to-surface (NTS) method. A summary of previous

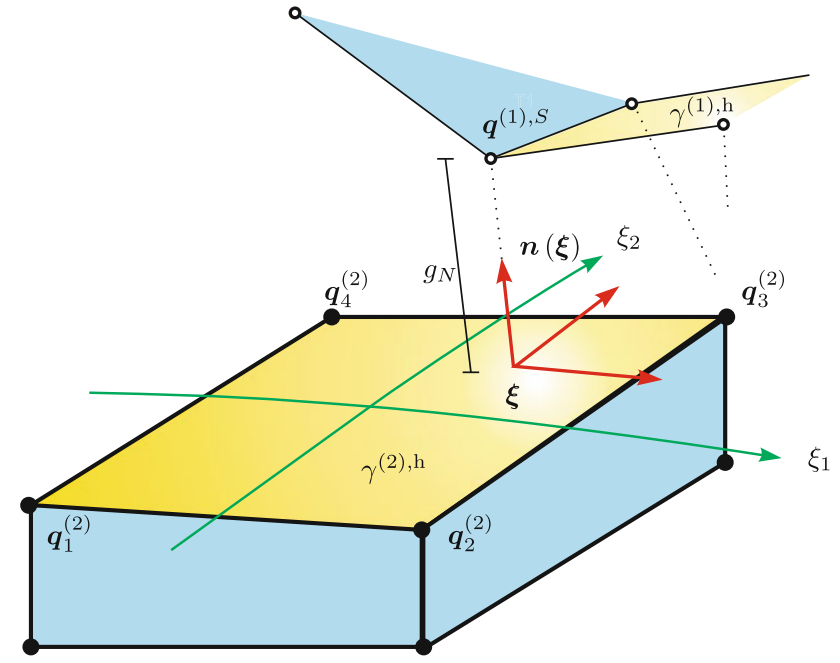

Fig. 2 Initial configuration of the representative NTS element: Closest point projection of the node $\boldsymbol{q}^{(1), S}$ onto the surface $\gamma^{(2), \mathrm{h}}$

developments in the context of the NTS method can be found in Laursen's [24] and Wriggers' [31] textbooks.

To illustrate the approach we consider a typical NTS element depicted in Fig. 2. This method constrains the system in such a way that the gap $g_{N}$ remains greater or equal zero. The gap rests on the closest point projection of a node $\boldsymbol{q}^{(1), S}$, which is part of the slave side $\gamma^{(1), \mathrm{h}}$, onto the opposing master side $\gamma^{(2), \mathrm{h}}$ and measures the distance between $\boldsymbol{q}^{(1), S}$ and the projected point, on $\gamma^{(2), h}$. Thus, a typical constraint function depends on the set of relevant vectors

$\eta_{S}=\left\{\overline{\boldsymbol{q}}_{I}^{S}\right\}=\left\{\boldsymbol{q}^{(1), S}, \boldsymbol{q}_{1}^{(2)}, \ldots, \boldsymbol{q}_{4}^{(2)}\right\}$

The closest point projection yields

$\boldsymbol{\varphi}^{(2), \mathrm{h}}(\boldsymbol{\xi}, t)=\sum_{C=1}^{4} \hat{N}^{C}(\boldsymbol{\xi}) \boldsymbol{q}_{c}^{(2)}(t)$,

where the local convective coordinates $\xi=\left(\xi_{1}, \xi_{2}\right)$ identify the projected point on $\gamma^{(2), \mathrm{h}}$ (see [21-23,32]). Since we use isoparametric tri-linear solid elements, $\hat{N}^{C}(\xi)$ are standard bi-linear local shape functions (see Fig. 2).

The new framework described in the following allows us to accomplish two major goals: The determination of the configuration dependent convective coordinates is simplified as does the construction of an energy-momentum scheme based on the notion of a discrete derivative in the sense of Gonzalez [11].

A measurement of the aforementioned distance between both surfaces can be established via the gap function $g_{N}^{S}(\boldsymbol{X}, t)$, given by

$g_{N}^{S}(\boldsymbol{X}, t)=\left(\boldsymbol{q}^{(1), S}-\sum_{C=1}^{4} \hat{N}^{C}(\boldsymbol{\xi}) \boldsymbol{q}_{C}^{(2)}\right) \cdot \boldsymbol{n}(\boldsymbol{\xi})$. 
Note, that the unit normal vector $\boldsymbol{n}(\boldsymbol{\xi})$ also depends on the convective coordinates. Now the impenetrability condition can be stated as

$\Phi^{S}\left(\overline{\boldsymbol{q}}_{I}^{S}\right)=g_{N}^{S}(\boldsymbol{X}, t) \leq 0$.

The corresponding nodal force vector $f^{\mathrm{c}, A}=\boldsymbol{f}^{(1), \mathrm{c}, A}+$ $f^{(2), c, A}$ can be written as (see 11)

$G^{c, \mathrm{~h}}(\boldsymbol{q}, \delta \boldsymbol{q})=\delta \boldsymbol{q}_{A} \cdot \boldsymbol{f}^{\mathrm{c}, A}=\delta \overline{\boldsymbol{q}}_{A}^{S} \cdot \lambda_{S} \nabla_{q_{\mathrm{A}}} \Phi^{S}$,

where $\lambda_{S}$ denotes the Lagrange multiplier and $\lambda_{S} \boldsymbol{n}(\boldsymbol{\xi})$ the corresponding traction in normal direction. Since we assume no adhesion, the tractions have to be positive, i.e. $\lambda_{S} \geq 0$. In combination with

$\lambda_{S}(\boldsymbol{X}, t) g_{N}^{S}(\boldsymbol{X}, t)=0$

we get the classical Kuhn-Tucker complementary conditions. These conditions separate the set of potential contact nodes $\bar{\omega}$ into an active set $\mathcal{A}$ and an inactive set $\mathcal{I}$, such that $\bar{\omega}=\mathcal{A} \cup \mathcal{I}$ and $\mathcal{A} \cap \mathcal{I}=\emptyset$. The discrete multiplier space can now be defined on the slave side as

$\mathcal{C}^{\mathrm{h}}=\left\{\lambda^{\mathrm{h}} \in C^{-1}\left(\gamma^{(1), \mathrm{h}}\right) \mid \lambda_{S}=0, \quad \forall \lambda_{S} \in \mathcal{I}\right\}$

with

$\lambda^{\mathrm{h}}=\sum_{A \in \bar{\omega}} N^{A}\left(\boldsymbol{X}_{S}\right) \lambda_{A}=\delta_{S}^{A} \lambda_{A}=\lambda_{S}$

where $\delta_{S}^{A}$ denotes the classical Kronecker delta and $\boldsymbol{X}_{S}^{(1)}$ a material point on $\Gamma^{(1), \mathrm{h}}$ which coincides with the node $\mathrm{S}$ on $\gamma^{(1), \mathrm{h}}$. Now we can define the total augmented potential energy as follows

$$
\begin{aligned}
V^{\text {aug }}(\boldsymbol{q})= & \sum_{i=1}^{2}\left(V^{(i), \text { int }}\left(\boldsymbol{q}^{(i)}\right)+V^{(i), \operatorname{ext}}\left(\boldsymbol{q}^{(i)}\right)\right) \\
& +\sum_{S \in \bar{\omega}} \lambda_{S} \Phi^{S}\left(\overline{\boldsymbol{q}}_{I}\right)
\end{aligned}
$$

so that the virtual work (11) reads

$G^{\mathrm{h}}(\boldsymbol{q}, \delta \boldsymbol{q})=\sum_{i=1}^{2} \delta \boldsymbol{q}_{A}^{(i)} \cdot\left[M^{A B} \ddot{\boldsymbol{q}}_{B}+\nabla_{\boldsymbol{q}_{\mathrm{A}}^{(\mathrm{i})}} V^{\mathrm{aug}}(\boldsymbol{q})\right]$

\section{Mixed formulation}

For our new approach we extend a specific coordinate augmentation technique originally introduced by Betsch and Uhlar [9] and adapted subsequently to domain decomposition problems in Hesch and Betsch [17]. The coordinate augmentation technique can be regarded as reducible formulation and classified as mixed method (see [35,36]). This technique leads to additional coordinates that can be appended to the set $\eta_{S}$ of original coordinates

$\eta_{S}^{\text {aug }}=\eta_{S} \cup\left\{\boldsymbol{d}^{S}\right\} \cup\left\{\boldsymbol{f}^{S}\right\}$

pertaining to the representative NTS element. In the present context the additional coordinates $\boldsymbol{d}^{S} \in \mathbb{R}^{3}$ play the role of the unit normal vector $\boldsymbol{n}$, whereas $\boldsymbol{f}^{S} \in \mathbb{R}^{2}$ stands for the convective coordinates $\xi$. To link the new coordinates to the original ones the following five additional constraint functions are introduced

$\boldsymbol{\Phi}^{\mathrm{aug}, S}\left(\overline{\boldsymbol{q}}^{S}, \boldsymbol{d}^{S}, \boldsymbol{f}^{S}\right)=\left[\begin{array}{c}\boldsymbol{d}^{S} \cdot \boldsymbol{a}_{1}\left(\boldsymbol{f}^{S}\right) \\ \boldsymbol{d}^{S} \cdot \boldsymbol{a}_{2}\left(\boldsymbol{f}^{S}\right) \\ \frac{1}{2}\left(\boldsymbol{d}^{S} \cdot \boldsymbol{d}^{S}-1\right) \\ \left(\boldsymbol{q}^{(1), S}-\sum_{C=1}^{4} \hat{N}^{C}\left(\boldsymbol{f}^{S}\right) \boldsymbol{q}_{C}^{(2)}\right) \cdot \boldsymbol{a}_{1}\left(\boldsymbol{f}^{S}\right) \\ \left(\boldsymbol{q}^{(1), S}-\sum_{C=1}^{4} \hat{N}^{C}\left(\boldsymbol{f}^{S}\right) \boldsymbol{q}_{C}^{(2)}\right) \cdot \boldsymbol{a}_{2}\left(\boldsymbol{f}^{S}\right)\end{array}\right]$

where $\boldsymbol{a}_{\alpha}$ denote the tangential vectors

$\boldsymbol{a}_{\alpha}(\boldsymbol{\xi})=\sum_{C=1}^{4} \hat{N}_{, \alpha}^{C}(\boldsymbol{\xi}) \boldsymbol{q}_{C}^{(2)}$

The modified NTS constraints can now be expressed exclusively in terms of the set $\eta_{S}^{\text {aug }}$

$\bar{\Phi}^{S}\left(\overline{\boldsymbol{q}}^{S}, \boldsymbol{d}^{S}, \boldsymbol{f}^{S}\right)=\left(\boldsymbol{q}^{(1), S}-\sum_{C=1}^{4} \hat{N}^{C}\left(\boldsymbol{f}^{S}\right) \boldsymbol{q}_{C}^{(2)}\right) \cdot \boldsymbol{d}^{S}$.

To simplify later developments, we collect the constraints (30) as well as the augmented constraints (28) and arrange them in a vector of constraint functions

$\boldsymbol{g}^{S}\left(\overline{\boldsymbol{q}}^{S}, \boldsymbol{d}^{S}, \boldsymbol{f}^{S}\right)=\left[\begin{array}{c}\bar{\Phi}^{S}\left(\overline{\boldsymbol{q}}^{S}, \boldsymbol{d}^{S}, \boldsymbol{f}^{S}\right) \\ \boldsymbol{\Phi}^{\text {aug }, S}\left(\overline{\boldsymbol{q}}^{S}, \boldsymbol{d}^{S}, \boldsymbol{f}^{S}\right)\end{array}\right]$.

\subsection{Fundamental properties of the mixed formulation}

For the verification of various conservation laws in the fully discrete setting (see Sect. 5.1) we need additional relations. In particular, we demonstrate the effects of rigid body transformations, which we need later on to proof algorithmic conservation of linear and angular momentum. Therefore, rigid motions of the form

$\overline{\boldsymbol{q}}_{I}^{S, \sharp}=\boldsymbol{c}+\boldsymbol{Q} \overline{\boldsymbol{q}}_{I}^{S}$

are considered. Here, $\boldsymbol{c} \in \mathbb{R}^{3}$ is a constant vector, and $\boldsymbol{Q} \in$ $S O(3)$ is a rotation tensor. It is easy to verify the following property 


$$
\begin{aligned}
& \boldsymbol{g}^{S}\left(\overline{\boldsymbol{q}}^{S, \sharp}, \boldsymbol{d}^{S}, \boldsymbol{f}^{S}\right)=\left[\begin{array}{c}
\left(\boldsymbol{q}^{(1), S}-\sum_{C=1}^{4} \hat{N}^{C} \boldsymbol{q}_{c}^{(2)}\right) \cdot \boldsymbol{Q}^{T} \boldsymbol{d}^{S} \\
\boldsymbol{a}_{1}\left(\boldsymbol{f}_{S}\right) \cdot \boldsymbol{Q}^{T} \boldsymbol{d}_{S} \\
\boldsymbol{a}_{2}\left(\boldsymbol{f}_{S}\right) \cdot \boldsymbol{Q}^{T} \boldsymbol{d}_{S} \\
\frac{1}{2}\left(\left(\boldsymbol{Q}^{T} \boldsymbol{d}_{S}\right) \cdot\left(\boldsymbol{Q}^{T} \boldsymbol{d}_{S}\right)-1\right) \\
\left(\boldsymbol{q}^{(1), S}-\sum_{C=1}^{4} \hat{N}^{C}\left(\boldsymbol{f}^{S}\right) \boldsymbol{q}_{c}^{(2)}\right) \cdot \boldsymbol{Q}^{T} \boldsymbol{Q} \boldsymbol{a}_{1}\left(\boldsymbol{f}_{S}\right) \\
\left(\boldsymbol{q}^{(1), S}-\sum_{C=1}^{4} \hat{N}^{C}\left(\boldsymbol{f}^{S}\right) \boldsymbol{q}_{c}^{(2)}\right) \cdot \boldsymbol{Q}^{T} \boldsymbol{Q} \boldsymbol{a}_{2}\left(\boldsymbol{f}_{S}\right)
\end{array}\right] \\
& =\boldsymbol{g}^{S}\left(\overline{\boldsymbol{q}}^{S}, \boldsymbol{Q}^{T} \boldsymbol{d}^{S}, \boldsymbol{f}^{S}\right)
\end{aligned}
$$

where the characteristics of the shape functions $\left(\sum_{A} N^{A}\right.$ $(\boldsymbol{X})=1)$ have been used. To show translational invariance we substitute $\boldsymbol{c}=\epsilon \boldsymbol{\mu}, \boldsymbol{\mu} \in \mathbb{R}^{3}$ and $\boldsymbol{Q}=\boldsymbol{I}$, where $\boldsymbol{I}$ denotes the identity matrix. Equation (33) implies, that

$\boldsymbol{g}^{S}\left(\overline{\boldsymbol{q}}^{S}+\epsilon \boldsymbol{\mu}, \boldsymbol{d}^{S}, \boldsymbol{f}^{S}\right)=\boldsymbol{g}^{S}\left(\overline{\boldsymbol{q}}^{S}, \boldsymbol{d}^{S}, \boldsymbol{f}^{S}\right)$

holds for arbitrary $\epsilon \in \mathbb{R}$. Consequently,

$\mathbf{0}=\left.\frac{\mathrm{d}}{\mathrm{d} \epsilon}\right|_{\epsilon=0} \boldsymbol{g}^{S}\left(\overline{\boldsymbol{q}}^{S}+\epsilon \boldsymbol{\mu}, \boldsymbol{d}^{S}, \boldsymbol{f}^{S}\right)=\sum_{I}\left(\partial_{\overline{\boldsymbol{q}}_{I}} \boldsymbol{g}^{S}\right) \cdot \boldsymbol{\mu}$

which proves translational invariance. Substituting $\boldsymbol{c}=\mathbf{0}$ and $\boldsymbol{Q}=\exp (\epsilon \hat{\boldsymbol{\mu}})$ into (33) yields

$\boldsymbol{g}^{S}\left(\exp (\epsilon \hat{\boldsymbol{\mu}}) \overline{\boldsymbol{q}}^{S}, \boldsymbol{d}^{S}, \boldsymbol{f}^{S}\right)-\boldsymbol{g}^{S}\left(\overline{\boldsymbol{q}}^{S}, \exp (-\epsilon \hat{\boldsymbol{\mu}}) \boldsymbol{d}^{S}, \boldsymbol{f}^{S}\right)=\mathbf{0}$

Here, $\exp (\epsilon \hat{\boldsymbol{\mu}}) \in S O(3)$ denotes the exponential map of a skew-symmetric tensor $\hat{\mu}$, which can be associated with an axial vector $\boldsymbol{\mu}$, so that $\hat{\boldsymbol{\mu}} \boldsymbol{a}=\boldsymbol{\mu} \times \boldsymbol{a}$ for any $\boldsymbol{a} \in \mathbb{R}^{3}$. Accordingly, we end up with

$$
\begin{aligned}
& \mathbf{0}=\left.\frac{\mathrm{d}}{\mathrm{d} \epsilon}\right|_{\epsilon=0}\left[\boldsymbol{g}^{S}\left(\exp (\epsilon \hat{\boldsymbol{\mu}}) \overline{\boldsymbol{q}}^{S}, \boldsymbol{d}^{S}, \boldsymbol{f}^{S}\right)\right. \\
& -\boldsymbol{g}^{S}\left(\overline{\boldsymbol{q}}^{S}, \exp (-\epsilon \hat{\boldsymbol{\mu}}) \boldsymbol{d}^{S}, \boldsymbol{f}^{S}\right)=\mathbf{0} \\
& \sum_{A}\left(\partial_{\overline{\boldsymbol{q}}_{A}^{S}} \boldsymbol{g}^{S}\right) \cdot \hat{\boldsymbol{\mu}} \overline{\boldsymbol{q}}_{A}^{S}+\left(\partial_{\boldsymbol{d}^{S}} \boldsymbol{g}^{S}\right) \cdot \hat{\boldsymbol{\mu}} \boldsymbol{d}^{S}=\mathbf{0} \\
& \sum_{A}\left(\partial_{\overline{\boldsymbol{q}}_{A}^{S}} \boldsymbol{g}^{S}\right) \cdot\left(\boldsymbol{\mu} \times \overline{\boldsymbol{q}}_{A}^{S}\right)+\left(\partial_{\boldsymbol{d}^{S}} \boldsymbol{g}^{S}\right) \cdot\left(\boldsymbol{\mu} \times \boldsymbol{d}^{S}\right)=\mathbf{0}
\end{aligned}
$$

for any vector $\mu \in \mathbb{R}^{3}$.

\subsection{Constraints in terms of invariants}

Translational and rotational invariance properties have to be in agreement with Cauchy's representation theorem, i.e. it has to be possible to rewrite the system in terms of appropriate invariants. In particular, we define three sets of invariants as follows ${ }^{1}$

$\mathbb{S}\left(\eta_{s}^{\text {aug }}\right)=\left\{\left(\overline{\boldsymbol{q}}_{I}^{S}-\boldsymbol{q}_{1}^{(2)}\right) \cdot\left(\overline{\boldsymbol{q}}_{J}^{S}-\boldsymbol{q}_{1}^{(2)}\right), 1 \leq I \leq J \leq 5\right\}$

$\tilde{\mathbb{S}}\left(\eta_{s}^{\text {aug }}\right)=\left\{\left(\overline{\boldsymbol{q}}_{I}^{S}-\boldsymbol{q}_{1}^{(2)}\right) \cdot \boldsymbol{d}^{S}, I=1, \ldots, 5\right\}$

$\overline{\mathbb{S}}\left(\eta_{s}^{\text {aug }}\right)=\left\{\boldsymbol{d}^{S} \cdot \boldsymbol{d}^{S}\right\}$

and establish a fourth set composed of the augmented coordinates $f^{S}$

$\hat{\mathbb{S}}\left(\eta_{s}^{\text {aug }}\right)=\left\{f_{\alpha}^{S}, \alpha=1,2\right\}$.

Next, we rewrite the modified NTS constraints in terms of the above given invariants as follows

$$
\begin{aligned}
\bar{\Phi}^{S}\left(\overline{\boldsymbol{q}}^{S}, \boldsymbol{d}_{S}, \boldsymbol{f}_{S}\right)= & \left(\boldsymbol{q}^{(1), S}-\boldsymbol{q}_{1}^{(2)}-\sum_{J} N^{J}\left(\boldsymbol{f}^{S}\right) \boldsymbol{q}_{J}^{(2)}\right. \\
& \left.+\sum_{J} N^{J}\left(\boldsymbol{f}^{S}\right) \boldsymbol{q}_{1}^{(2)}\right) \cdot \boldsymbol{d}^{S} \\
= & \left(\boldsymbol{q}^{(1), S}-\boldsymbol{q}_{1}^{(2)}\right) \cdot \boldsymbol{d}^{S} \\
& -\sum_{J=2}^{4} N^{J}\left(\boldsymbol{f}^{S}\right)\left(\boldsymbol{q}_{J}^{(2)}-\boldsymbol{q}_{1}^{(2)}\right) \cdot \boldsymbol{d}^{S}
\end{aligned}
$$

Then we introduce a vector of invariants

$\overline{\boldsymbol{\pi}}\left(\overline{\boldsymbol{q}}^{S}, \boldsymbol{d}^{S}, \boldsymbol{f}^{S}\right)=\left[\begin{array}{c}\pi_{1} \\ \pi_{2} \\ \pi_{3} \\ \pi_{4} \\ \pi_{5} \\ \pi_{6}\end{array}\right]=\left[\begin{array}{c}\left(\boldsymbol{q}^{(1), S}-\boldsymbol{q}_{1}^{(2)}\right) \cdot \boldsymbol{d}^{S} \\ \left(\boldsymbol{q}_{2}^{(2)}-\boldsymbol{q}_{1}^{(2)}\right) \cdot \boldsymbol{d}^{S} \\ \left(\boldsymbol{q}_{3}^{(2)}-\boldsymbol{q}_{1}^{(2)}\right) \cdot \boldsymbol{d}^{S} \\ \left(\boldsymbol{q}_{4}^{(2)}-\boldsymbol{q}_{1}^{(2)}\right) \cdot \boldsymbol{d}^{S} \\ f_{1}^{S} \\ f_{2}^{S}\end{array}\right]$

and rewrite the modified NTS constraints (30) in the form

$\tilde{\Phi}^{S}\left(\overline{\boldsymbol{\pi}}\left(\overline{\boldsymbol{q}}^{S}, \boldsymbol{d}^{S}, \boldsymbol{f}^{S}\right)\right)=\pi_{1}-\sum_{I=2}^{4} N_{I}\left(\pi_{5}, \pi_{6}\right) \pi_{I}$

Note that the augmented coordinates $f_{1}^{S}$ and $f_{2}^{S}$ play the role of linear invariants in (41). They do not depend explicitly on the configuration and the constraints $(28)_{4,5}$ used to calculate them are invariant as shown in (33). Following the same ideas, the additional constraints (28) can be written as

\footnotetext{
$\overline{1}$ The invariance property of the last two sets can only be established, if the augmented constraints (33) are fulfilled.
} 


$$
\tilde{\boldsymbol{\Phi}}^{\text {aug }}\left(\boldsymbol{\pi}\left(\overline{\boldsymbol{q}}^{S}, \boldsymbol{d}^{S}, \boldsymbol{f}^{S}\right)\right)=\left[\begin{array}{c}
\sum_{I=2}^{4} N_{I, \pi_{5}}\left(\pi_{5}, \pi_{6}\right) \pi_{I} \\
\sum_{I=2}^{4} N_{I, \pi_{6}}\left(\pi_{5}, \pi_{6}\right) \pi_{I} \\
\frac{1}{2}\left(\pi_{7}-1\right) \\
\sum_{I=2}^{4} N_{I, \pi_{5}}\left(\pi_{5}, \pi_{6}\right) \pi_{I+6}-K_{1} \\
\sum_{I=2}^{4} N_{I, \pi_{6}}\left(\pi_{5}, \pi_{6}\right) \pi_{I+6}-K_{2}
\end{array}\right]
$$

with

$$
\begin{aligned}
& K_{1}=\sum_{I=2}^{4} \sum_{J=2}^{4} N_{I, \pi_{5}}\left(\pi_{5}, \pi_{6}\right) N_{J}\left(\pi_{5}, \pi_{6}\right)\left(\boldsymbol{q}_{I}^{(2)}-\boldsymbol{q}_{1}^{(2)}\right) \cdot\left(\boldsymbol{q}_{J}^{(2)}-\boldsymbol{q}_{1}^{(2)}\right) \\
& K_{2}=\sum_{I=2}^{4} \sum_{J=2}^{4} N_{I, \pi_{6}}\left(\pi_{5}, \pi_{6}\right) N_{J}\left(\pi_{5}, \pi_{6}\right)\left(\boldsymbol{q}_{I}^{(2)}-\boldsymbol{q}_{1}^{(2)}\right) \cdot\left(\boldsymbol{q}_{J}^{(2)}-\boldsymbol{q}_{1}^{(2)}\right)
\end{aligned}
$$

This gives rise to the definition of the extended vector of invariants

$\pi\left(\overline{\boldsymbol{q}}^{S}, \boldsymbol{d}^{S}, \boldsymbol{f}^{S}\right)=\left[\begin{array}{c}\overline{\boldsymbol{\pi}} \\ \pi_{7} \\ \pi_{8} \\ \pi_{9} \\ \pi_{10} \\ \pi_{11} \\ \pi_{12} \\ \pi_{13} \\ \pi_{14} \\ \pi_{15} \\ \pi_{16}\end{array}\right]=\left[\begin{array}{c}\boldsymbol{d}^{S} \cdot \boldsymbol{d}^{S} \\ \left(\boldsymbol{q}_{2}^{(2)}-\boldsymbol{q}_{1}^{(2)}\right) \cdot\left(\boldsymbol{q}_{S}^{(1)}-\boldsymbol{q}_{1}^{(2)}\right) \\ \left(\boldsymbol{q}_{3}^{(2)}-\boldsymbol{q}_{1}^{(2)}\right) \cdot\left(\boldsymbol{q}_{S}^{(1)}-\boldsymbol{q}_{1}^{(2)}\right) \\ \left(\boldsymbol{q}_{4}^{(2)}-\boldsymbol{q}_{1}^{(2)}\right) \cdot\left(\boldsymbol{q}_{S}^{(1)}-\boldsymbol{q}_{1}^{(2)}\right) \\ \left(\boldsymbol{q}_{2}^{(2)}-\boldsymbol{q}_{1}^{(2)}\right) \cdot\left(\boldsymbol{q}_{2}^{(2)}-\boldsymbol{q}_{1}^{(2)}\right) \\ \left(\boldsymbol{q}_{2}^{(2)}-\boldsymbol{q}_{1}^{(2)}\right) \cdot\left(\boldsymbol{q}_{3}^{(2)}-\boldsymbol{q}_{1}^{(2)}\right) \\ \left(\boldsymbol{q}_{2}^{(2)}-\boldsymbol{q}_{1}^{(2)}\right) \cdot\left(\boldsymbol{q}_{4}^{(2)}-\boldsymbol{q}_{1}^{(2)}\right) \\ \left(\boldsymbol{q}_{3}^{(2)}-\boldsymbol{q}_{1}^{(2)}\right) \cdot\left(\boldsymbol{q}_{3}^{(2)}-\boldsymbol{q}_{1}^{(2)}\right) \\ \left(\boldsymbol{q}_{3}^{(2)}-\boldsymbol{q}_{1}^{(2)}\right) \cdot\left(\boldsymbol{q}_{4}^{(2)}-\boldsymbol{q}_{1}^{(2)}\right) \\ \left(\boldsymbol{q}_{4}^{(2)}-\boldsymbol{q}_{1}^{(2)}\right) \cdot\left(\boldsymbol{q}_{4}^{(2)}-\boldsymbol{q}_{1}^{(2)}\right)\end{array}\right]$

At last, we can write

$$
\tilde{\boldsymbol{g}}^{S}\left(\boldsymbol{\pi}\left(\overline{\boldsymbol{q}}^{S}, \boldsymbol{d}^{S}, \boldsymbol{f}^{S}\right)\right)=\boldsymbol{g}^{S}\left(\overline{\boldsymbol{q}}^{S}, \boldsymbol{d}^{S}, \boldsymbol{f}^{S}\right)
$$

Of course, the fundamental properties derived in Sect. 4.1 are retained by the reformulated constraints. With regard to (35) we can state that

$$
\mathbf{0}=\left.\frac{\mathrm{d}}{\mathrm{d} \epsilon}\right|_{\epsilon=0} \tilde{\boldsymbol{g}}^{S}\left(\boldsymbol{\pi}\left(\overline{\boldsymbol{q}}^{S}+\epsilon \boldsymbol{\mu}, \boldsymbol{d}^{S}, \boldsymbol{f}^{S}\right)\right)=\partial_{\pi} \tilde{\boldsymbol{g}}^{S} \cdot \sum_{A}\left(\partial_{\overline{\boldsymbol{q}}_{A}} \boldsymbol{\pi}\right) \cdot \boldsymbol{\mu}
$$

which is true a priori due to the construction of the vector of invariants. For the rotational part in (37) follows with regard to property (33) and (36)

$$
\begin{aligned}
& \mathbf{0}=\left.\frac{\mathrm{d}}{\mathrm{d} \epsilon}\right|_{\epsilon=0}\left[\tilde{\boldsymbol{g}}^{S}\left(\boldsymbol{\pi}\left(\exp (\hat{\boldsymbol{\mu}}) \overline{\boldsymbol{q}}^{S}, \boldsymbol{d}^{S}, \boldsymbol{f}^{S}\right)\right)\right. \\
& -\tilde{\boldsymbol{g}}^{S}\left(\boldsymbol{\pi}\left(\overline{\boldsymbol{q}}^{S}, \exp (-\epsilon \hat{\boldsymbol{\mu}}) \boldsymbol{d}^{S}, \boldsymbol{f}^{S}\right)\right)=\mathbf{0}
\end{aligned}
$$

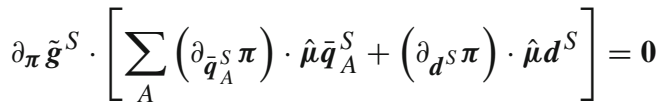

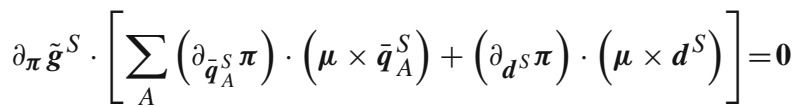

which is also true a priori due to the construction of the vector of invariants.

\section{Equations of motion}

The finite-dimensional, semi-discrete system at hand can be associated with an augmented Lagrangian of the form

$$
L_{\lambda}=\frac{1}{2} \dot{\boldsymbol{q}} \cdot \boldsymbol{M} \dot{\boldsymbol{q}}-V(\boldsymbol{q})-\sum_{S \in \bar{\omega}} \tilde{\boldsymbol{g}}^{S}\left(\boldsymbol{\pi}\left(\overline{\boldsymbol{q}}^{S}, \boldsymbol{d}^{S}, \boldsymbol{f}^{S}\right)\right) \cdot \lambda_{S}
$$

where $\lambda_{S}$ are the corresponding multipliers of the augmented set of constraints. Then the equations of motion for the constrained system under consideration take the form (cf. [17])

$$
\begin{aligned}
\boldsymbol{M} \ddot{\boldsymbol{q}} & =-\nabla V(\boldsymbol{q})-\sum_{S \in \bar{\omega}}\left(D_{1} \boldsymbol{\pi}\left(\overline{\boldsymbol{q}}^{S}, \boldsymbol{d}^{S}, \boldsymbol{f}^{S}\right)\right)^{T} \nabla_{\pi} \tilde{\boldsymbol{g}}^{S}(\boldsymbol{\pi}) \cdot \lambda_{S} \\
\mathbf{0} & =\sum_{S \in \bar{\omega}}\left(D_{2} \boldsymbol{\pi}\left(\overline{\boldsymbol{q}}^{S}, \boldsymbol{d}^{S}, \boldsymbol{f}^{S}\right)\right)^{T} \nabla_{\pi} \tilde{\boldsymbol{g}}^{S}(\boldsymbol{\pi}) \cdot \lambda_{S} \\
\mathbf{0} & =\sum_{S \in \bar{\omega}}\left(D_{3} \boldsymbol{\pi}\left(\overline{\boldsymbol{q}}^{S}, \boldsymbol{d}^{S}, \boldsymbol{f}^{S}\right)\right)^{T} \nabla_{\boldsymbol{\pi}} \tilde{\boldsymbol{g}}^{S}(\boldsymbol{\pi}) \cdot \lambda_{S} \\
\mathbf{0} & =\left[\begin{array}{c}
\tilde{\boldsymbol{g}}^{S=1}\left(\boldsymbol{\pi}\left(\overline{\boldsymbol{q}}^{S}, \boldsymbol{d}^{S}, \boldsymbol{f}^{S}\right)\right) \\
\vdots \\
\tilde{\boldsymbol{g}}^{S=n_{\text {surf }}}\left(\boldsymbol{\pi}\left(\overline{\boldsymbol{q}}^{S}, \boldsymbol{d}^{S}, \boldsymbol{f}^{S}\right)\right)
\end{array}\right]
\end{aligned}
$$

For the semi-discrete system at hand we have to verify conservation of linear and angular momentum as well as total energy. We omit the verification of conservation of linear momentum due to the translational invariance of the constraints (47) and demonstrate exemplarily the verification of conservation of angular momentum

$\boldsymbol{J}=\sum_{A, B} M^{A B} \boldsymbol{q}_{A} \times \dot{\boldsymbol{q}}_{B}$

where we assume for simplicity the absence of external forces. In particular, we assume that $V=0$, since only the modified constraints are of interest. For an arbitrary vector $\boldsymbol{\mu}$ follows 


$$
\begin{aligned}
\boldsymbol{\mu} \cdot \frac{d}{d t} \boldsymbol{J} & =\boldsymbol{\mu} \cdot \sum_{A, B} M^{A B} \boldsymbol{q}_{A} \times \ddot{\boldsymbol{q}}_{B} \\
& =-\boldsymbol{\mu} \cdot \sum_{A} \boldsymbol{q}_{A} \times \sum_{S \in \bar{\omega}}\left(\partial_{\boldsymbol{q}_{A}} \boldsymbol{\pi}\left(\overline{\boldsymbol{q}}^{S}, \boldsymbol{d}^{S}, \boldsymbol{f}^{S}\right)\right)^{T} \nabla_{\boldsymbol{\pi}} \tilde{\boldsymbol{g}}^{S}(\boldsymbol{\pi}) \cdot \lambda_{S} \\
& =\sum_{S \in \bar{\omega}} \lambda_{S} \cdot D \tilde{\boldsymbol{g}}^{S}(\boldsymbol{\pi}) \cdot \sum_{A}\left(\partial_{\boldsymbol{q}_{A}} \boldsymbol{\pi}\left(\overline{\boldsymbol{q}}^{S}, \boldsymbol{d}^{S}, \boldsymbol{f}^{S}\right)\right) \cdot \hat{\boldsymbol{q}}_{A} \boldsymbol{\mu}
\end{aligned}
$$

With regard to (48) it is easy to show that

$$
\begin{array}{r}
D \tilde{\boldsymbol{g}}^{S}(\boldsymbol{\pi}) \cdot \sum_{A}\left(\partial_{\boldsymbol{q}_{A}} \boldsymbol{\pi}\left(\overline{\boldsymbol{q}}^{S}, \boldsymbol{d}^{S}, \boldsymbol{f}^{S}\right)\right) \cdot \hat{\boldsymbol{q}}_{A} \boldsymbol{\mu} \\
=-D \tilde{\boldsymbol{g}}^{S}(\boldsymbol{\pi}) \cdot\left(\partial_{\boldsymbol{d}} \boldsymbol{\pi}\left(\overline{\boldsymbol{q}}^{S}, \boldsymbol{d}^{S}, \boldsymbol{f}^{S}\right)\right) \cdot \hat{\boldsymbol{d}}_{S} \boldsymbol{\mu}
\end{array}
$$

Insertion into (52) yields

$$
\begin{aligned}
& \boldsymbol{\mu} \cdot \frac{d}{d t} \boldsymbol{J}=-\sum_{S \in \bar{\omega}} \lambda_{S} \cdot D \tilde{\boldsymbol{g}}^{S}(\boldsymbol{\pi}) \cdot\left(\partial_{\boldsymbol{d}} S \boldsymbol{\pi}\left(\overline{\boldsymbol{q}}^{S}, \boldsymbol{d}^{S}, \boldsymbol{f}^{S}\right)\right) \cdot \hat{\boldsymbol{d}}_{S} \boldsymbol{\mu} \\
& =\boldsymbol{\mu} \cdot \sum_{S \in \bar{\omega}} \boldsymbol{d}_{S} \times\left(\partial_{\boldsymbol{d}^{S}} \boldsymbol{\pi}\left(\overline{\boldsymbol{q}}^{S}, \boldsymbol{d}^{S}, \boldsymbol{f}^{S}\right)\right)^{T} \nabla_{\pi} \tilde{\boldsymbol{g}}^{S}(\boldsymbol{\pi}) \cdot \lambda_{S} \\
& =0
\end{aligned}
$$

where use has been made of $(50)_{2}$. Since the mechanical system at hand is conservative, it is essential to verify conservation of energy for the semidiscrete, augmented system. Scalar multiplication of $(50)_{1}$ with $\dot{\boldsymbol{q}}$ yields

$$
\begin{aligned}
& \dot{\boldsymbol{q}} \cdot \boldsymbol{M} \ddot{\boldsymbol{q}}+\dot{\boldsymbol{q}} \cdot \nabla V(\boldsymbol{q}) \\
&+\dot{\boldsymbol{q}} \cdot \sum_{S \in \bar{\omega}}\left(D_{1} \boldsymbol{\pi}\left(\overline{\boldsymbol{q}}^{S}, \boldsymbol{d}^{S}, \boldsymbol{f}^{S}\right)\right)^{T} \nabla_{\pi} \tilde{\boldsymbol{g}}^{S}(\boldsymbol{\pi}) \cdot \lambda_{S}=0 \\
& \frac{d}{d t}\left(\frac{1}{2} \dot{\boldsymbol{q}} \cdot \boldsymbol{M} \dot{\boldsymbol{q}}\right)+\frac{d}{d t} V(\boldsymbol{q}) \\
& \quad+\sum_{S \in \bar{\omega}} \lambda_{S} \cdot D \tilde{\boldsymbol{g}}^{S}(\boldsymbol{\pi})\left(D_{1} \boldsymbol{\pi}\left(\overline{\boldsymbol{q}}^{S}, \boldsymbol{d}^{S}, \boldsymbol{f}^{S}\right)\right) \cdot \dot{\overline{\boldsymbol{q}}}^{S}=0
\end{aligned}
$$

Taking into account the consistency condition

$$
\begin{aligned}
& D \tilde{\boldsymbol{g}}^{S}(\boldsymbol{\pi}) \cdot\left(D_{1} \boldsymbol{\pi}\left(\overline{\boldsymbol{q}}^{S}, \boldsymbol{d}^{S}, \boldsymbol{f}^{S}\right)\right) \cdot \dot{\overline{\boldsymbol{q}}}^{S} \\
& \quad+D \tilde{\boldsymbol{g}}^{S}(\boldsymbol{\pi}) \cdot\left(D_{2} \boldsymbol{\pi}\left(\overline{\boldsymbol{q}}^{S}, \boldsymbol{d}^{S}, \boldsymbol{f}^{S}\right)\right) \cdot \dot{\boldsymbol{d}}^{S} \\
& \quad+D \tilde{\boldsymbol{g}}^{S}(\boldsymbol{\pi}) \cdot\left(D_{3} \boldsymbol{\pi}\left(\overline{\boldsymbol{q}}^{S}, \boldsymbol{d}^{S}, \boldsymbol{f}^{S}\right)\right) \cdot \dot{\boldsymbol{f}}^{S}=\mathbf{0}
\end{aligned}
$$

we can rewrite $\left(55_{2}\right)$ with regard to $(50)_{2}$ and $(50)_{3}$ as follows

$$
\begin{aligned}
\frac{d}{d t}(T+V)- & \sum_{S \in \bar{\omega}} \lambda_{S} \cdot\left[D \tilde{\boldsymbol{g}}^{S}(\boldsymbol{\pi}) \cdot\left(D_{2} \boldsymbol{\pi}\left(\overline{\boldsymbol{q}}^{S}, \boldsymbol{d}^{S}, \boldsymbol{f}^{S}\right)\right) \cdot \dot{\boldsymbol{d}}^{S}\right. \\
& \left.+D \tilde{\boldsymbol{g}}^{S}(\boldsymbol{\pi}) \cdot\left(D_{3} \boldsymbol{\pi}\left(\overline{\boldsymbol{q}}^{S}, \boldsymbol{d}^{S}, \boldsymbol{f}^{S}\right)\right) \cdot \dot{\boldsymbol{f}}^{S}\right]=0 \\
\frac{d}{d t}(T+V)= & 0
\end{aligned}
$$

Hence, the total system energy, defined as the sum of the kinetic energy $T=\frac{1}{2} \dot{\boldsymbol{q}} \cdot \boldsymbol{M} \dot{\boldsymbol{q}}$ and the potential energy, i.e.
$E(\boldsymbol{q}, \dot{\boldsymbol{q}})=\frac{1}{2} \dot{\boldsymbol{q}} \cdot \boldsymbol{M} \dot{\boldsymbol{q}}+V(\boldsymbol{q})$

is conserved.

\subsection{Time discretization}

The goal of this section is to perform an energy and momentum conserving time discretization of the semidiscrete system at hand. Therefore we subdivide the time interval of interest $t \in \mathbb{I}=[0, T]$ into partitions $\left[t_{n}, t_{n+1}\right]$ of constant length $\Delta t$ and apply a one step time integration scheme, similar to the energy-momentum schemes for constrained mechanical systems developed by Gonzalez [11], see also Betsch and Steinmann [8]. This is in contrast to the established energy-momentum schemes developed for unilateral contact constraints. In Laursen and Chawla [25] (and similarly in [1]) an algorithmic gap rate has been defined in exchange of the original constraints $(50)_{4}$. This ensures conservation of energy as well as conservation of the momentum maps by sacrificing the exact fulfillment of the constraints. In Laursen and Love [26] a velocity update procedure has been proposed to ensure the conservation properties. In contrast to the algorithmic gap rate approach as well as the method proposed in this paper the velocity update algorithm can display only first-order accuracy in time (see also [29]). Within our approach, the fully discretized equations of motion are given by

$$
\begin{aligned}
& \boldsymbol{q}_{n+1}-\boldsymbol{q}_{n}=\Delta t \boldsymbol{v}_{n+\frac{1}{2}} \\
& \boldsymbol{M}\left(\boldsymbol{v}_{n+1}-\boldsymbol{v}_{n}\right)=-\Delta t \bar{\nabla} V\left(\boldsymbol{q}_{n}, \boldsymbol{q}_{n+1}\right) \\
& -\Delta t \sum_{S \in \bar{\omega}}\left(D_{1} \pi\left(\overline{\boldsymbol{q}}_{n+\frac{1}{2}}^{S}, \boldsymbol{d}_{n+\frac{1}{2}}^{S}, \boldsymbol{f}_{n+\frac{1}{2}}^{S}\right)\right)^{T} \overline{\bar{\nabla}}_{\pi} \\
& \times \tilde{\boldsymbol{g}}^{S}\left(\boldsymbol{\pi}_{n}, \boldsymbol{\pi}_{n+1}\right) \cdot \lambda_{S, n+1} \\
& \mathbf{0}=\sum_{S \in \bar{\omega}}\left(D_{2} \pi\left(\overline{\boldsymbol{q}}_{n+\frac{1}{2}}^{S}, \boldsymbol{d}_{n+\frac{1}{2}}^{S}, \boldsymbol{f}_{n+\frac{1}{2}}^{S}\right)\right)^{T} \overline{\bar{\nabla}}_{\pi} \\
& \times \tilde{\boldsymbol{g}}^{S}\left(\boldsymbol{\pi}_{n}, \boldsymbol{\pi}_{n+1}\right) \cdot \lambda_{S, n+1} \\
& \mathbf{0}=\sum_{S \in \bar{\omega}}\left(D_{3} \pi\left(\overline{\boldsymbol{q}}_{n+\frac{1}{2}}^{S}, \boldsymbol{d}_{n+\frac{1}{2}}^{S}, \boldsymbol{f}_{n+\frac{1}{2}}^{S}\right)\right)^{T} \overline{\bar{\nabla}}_{\pi} \\
& \times \tilde{\boldsymbol{g}}^{S}\left(\boldsymbol{\pi}_{n}, \boldsymbol{\pi}_{n+1}\right) \cdot \lambda_{S, n+1} \\
& \mathbf{0}=\left[\begin{array}{c}
\tilde{\boldsymbol{g}}^{S=1}\left(\boldsymbol{\pi}\left(\overline{\boldsymbol{q}}_{n+1}^{S}, \boldsymbol{d}_{n+1}^{S}, \boldsymbol{f}_{n+1}^{S}\right)\right) \\
\vdots \\
\tilde{\boldsymbol{g}}^{S=n_{\text {surf }}}\left(\boldsymbol{\pi}\left(\overline{\boldsymbol{q}}_{n+1}^{S}, \boldsymbol{d}_{n+1}^{S}, \boldsymbol{f}_{n+1}^{S}\right)\right)
\end{array}\right]
\end{aligned}
$$

Here, the discrete gradient of the internal energy (in absence of additional external energy) $\bar{\nabla} V\left(\boldsymbol{q}_{n}, \boldsymbol{q}_{n+1}\right)$ facilitates conservation of energy and both momentum maps by design. We refer to Betsch and Steinmann [7] and the references therein for further details of energy-momentum conserving 
schemes in the context of non-linear elastodynamics. Furthermore, $\overline{\bar{\nabla}}_{\pi} \tilde{\boldsymbol{g}}^{S}\left(\boldsymbol{\pi}_{n}, \boldsymbol{\pi}_{n+1}\right)$ denotes a discrete gradient of the constraint functions, defined as (see [10])

$$
\begin{aligned}
& \overline{\bar{\nabla}}_{\pi} \tilde{\boldsymbol{g}}^{S}\left(\boldsymbol{\pi}_{n}, \boldsymbol{\pi}_{n+1}\right)=\nabla_{\pi} \tilde{\boldsymbol{g}}^{S}\left(\boldsymbol{\pi}_{n+\frac{1}{2}}\right) \\
& +\frac{\tilde{\boldsymbol{g}}^{S}\left(\boldsymbol{\pi}_{n+1}\right)-\tilde{\boldsymbol{g}}^{S}\left(\boldsymbol{\pi}_{n}\right)+\nabla_{\pi} \tilde{\boldsymbol{g}}^{S}\left(\boldsymbol{\pi}_{n+\frac{1}{2}}\right) \cdot \Delta \boldsymbol{\pi}}{\|\Delta \boldsymbol{\pi}\|^{2}} \Delta \boldsymbol{\pi}
\end{aligned}
$$

In this connection, the abbreviations $\Delta \pi=\pi_{n+1}-\pi_{n}, \pi_{n}=$ $\boldsymbol{\pi}\left(\overline{\boldsymbol{q}}_{I, n}^{S}, \boldsymbol{d}_{n}^{S}, \boldsymbol{f}_{n}^{S}\right), \boldsymbol{\pi}_{n+1}=\boldsymbol{\pi}\left(\overline{\boldsymbol{q}}_{I, n+1}^{S}, \boldsymbol{d}_{n+1}^{S}, \boldsymbol{f}_{n+1}^{S}\right)$, and $(\bullet)_{n+\frac{1}{2}}=\left((\bullet)_{n}+(\bullet)_{n+1}\right) / 2$ have been employed. Analogous to the time-continuous case, we start with the verification of the algorithmic conservation of angular momentum. Since the angular momentum map $\boldsymbol{J}(\boldsymbol{q}, \boldsymbol{v})$ is a quadratic function of $(\boldsymbol{q}, \boldsymbol{v})$ we can state that

$$
\begin{aligned}
& \boldsymbol{J}_{n+1}-\boldsymbol{J}_{n} \\
& =\left(D_{1} \boldsymbol{J}\left(\boldsymbol{q}_{n+\frac{1}{2}}, \boldsymbol{v}_{n+\frac{1}{2}}\right)\right)\left(\boldsymbol{q}_{n+1}-\boldsymbol{q}_{n}\right)+\left(D_{2} \boldsymbol{J}\left(\boldsymbol{q}_{n+\frac{1}{2}}, \boldsymbol{v}_{n+\frac{1}{2}}\right)\right) \\
& \quad \times\left(\boldsymbol{v}_{n+1}-\boldsymbol{v}_{n}\right)=-\sum_{A, B} M^{A B} \boldsymbol{v}_{B_{n+\frac{1}{2}}} \times\left(\boldsymbol{q}_{A_{n+1}}-\boldsymbol{q}_{A_{n}}\right) \\
& \quad+\sum_{A, B} M^{A B} \boldsymbol{q}_{B_{n+\frac{1}{2}}} \times\left(\boldsymbol{v}_{A_{n+1}}-\boldsymbol{v}_{A_{n}}\right)
\end{aligned}
$$

Scalar multiplication of the last equation with an arbitrary vector $\boldsymbol{\mu}$ and subsequently substituting from (59) $)_{1}$ and (59) yields

$$
\begin{aligned}
& \boldsymbol{\mu} \cdot\left(\boldsymbol{J}_{n+1}-\boldsymbol{J}_{n}\right)=-\Delta t \boldsymbol{\mu} \cdot \sum_{B} \boldsymbol{q}_{B_{n+\frac{1}{2}}} \\
& \times\left(\sum_{S \in \bar{\omega}}\left(\partial_{\boldsymbol{q}_{B}} \boldsymbol{\pi}\left(\overline{\boldsymbol{q}}_{n+\frac{1}{2}}^{S}, \boldsymbol{d}_{n+\frac{1}{2}}^{S}, \boldsymbol{f}_{n+\frac{1}{2}}^{S}\right)\right)^{T}\right. \\
& \left.\overline{\bar{\nabla}}_{\pi} \tilde{\boldsymbol{g}}^{S}\left(\boldsymbol{\pi}_{n}, \boldsymbol{\pi}_{n+1}\right) \cdot \lambda_{S, n+1}\right) \\
& =\Delta t \sum_{S \in \bar{\omega}} \lambda_{S, n+1} \cdot \sum_{B}\left(\partial_{\boldsymbol{q}_{B}} \boldsymbol{\pi}\left(\overline{\boldsymbol{q}}_{n+\frac{1}{2}}^{S}, \boldsymbol{d}_{n+\frac{1}{2}}^{S}, \boldsymbol{f}_{n+\frac{1}{2}}^{S}\right)\right)^{T} \\
& \overline{\bar{\nabla}}_{\pi} \tilde{\boldsymbol{g}}^{S}\left(\boldsymbol{\pi}_{n}, \boldsymbol{\pi}_{n+1}\right) \cdot \widehat{\boldsymbol{q}}_{B, n+\frac{1}{2}} \boldsymbol{\mu}
\end{aligned}
$$

Again, we incorporate the relation (48)

$$
\begin{aligned}
& \sum_{A}\left(\partial_{\boldsymbol{q}_{A}} \boldsymbol{\pi}\left(\overline{\boldsymbol{q}}_{n+\frac{1}{2}}^{S}, \boldsymbol{d}_{n+\frac{1}{2}}^{S}, \boldsymbol{f}_{n+\frac{1}{2}}^{S}\right)\right)^{T} \\
& \overline{\bar{\nabla}}_{\pi} \tilde{\boldsymbol{g}}^{S}\left(\boldsymbol{\pi}_{n}, \boldsymbol{\pi}_{n+1}\right) \cdot \hat{\boldsymbol{q}}_{A, n+\frac{1}{2}} \boldsymbol{\mu} \\
& +\partial_{\boldsymbol{d}} S \boldsymbol{\pi}\left(\overline{\boldsymbol{q}}_{n+\frac{1}{2}}^{S}, \boldsymbol{d}_{n+\frac{1}{2}}^{S}, \boldsymbol{f}_{n+\frac{1}{2}}^{S}\right)^{T} \\
& \overline{\bar{\nabla}}_{\pi} \tilde{\boldsymbol{g}}^{S}\left(\boldsymbol{\pi}_{n}, \boldsymbol{\pi}_{n+1}\right) \cdot \hat{\boldsymbol{d}}_{n+\frac{1}{2}}^{S} \boldsymbol{\mu}=0
\end{aligned}
$$

into the last equation

$$
\begin{aligned}
& \boldsymbol{\mu} \cdot\left(\boldsymbol{J}_{n+1}-\boldsymbol{J}_{n}\right) \\
& \left.=-\Delta t \sum_{S \in \bar{\omega}} \lambda_{S, n+1} \cdot \partial_{\boldsymbol{d}^{S}} \boldsymbol{\pi}\left(\overline{\boldsymbol{q}}_{n+\frac{1}{2}}^{S}, \boldsymbol{d}_{n+\frac{1}{2}}^{S}, \boldsymbol{f}_{n+\frac{1}{2}}^{S}\right)\right)^{T} \overline{\bar{\nabla}}_{\pi} \\
& \times \tilde{\boldsymbol{g}}^{S}\left(\boldsymbol{\pi}_{n}, \boldsymbol{\pi}_{n+1}\right) \cdot \widehat{\boldsymbol{d}}_{n+\frac{1}{2}}^{S} \boldsymbol{\mu} \\
& \left.=\Delta t \boldsymbol{\mu} \cdot \sum_{S \in \bar{\omega}} \boldsymbol{d}_{n+\frac{1}{2}}^{S} \times \partial_{\boldsymbol{d}} S \boldsymbol{\pi}\left(\overline{\boldsymbol{q}}_{I, n+\frac{1}{2}}^{S}, \boldsymbol{d}_{n+\frac{1}{2}}^{S}, \boldsymbol{f}_{n+\frac{1}{2}}^{S}\right)\right)^{T} \overline{\bar{\nabla}}_{\pi} \\
& \times \tilde{\boldsymbol{g}}^{S}\left(\boldsymbol{\pi}_{n}, \boldsymbol{\pi}_{n+1}\right) \cdot \lambda_{S, n+1} \\
& =0
\end{aligned}
$$

Eventually, we verify algorithmic conservation of energy. Two sources for the lack of energy conservation have to be considered: (a) The nonlinearity of the constraints and (b) the discretization error in time due to the active set strategy (cf. [16]). We will deal with the former first and then explain solution strategies for the latter at the end of this section.

For the verification of algorithmic conservation of energy we have to show that the net power input to the system within each time step is zero. Scalar multiplication of $(59)_{2}$ with $v_{n+\frac{1}{2}}$ yields

$$
\begin{gathered}
\boldsymbol{v}_{n+\frac{1}{2}} \cdot \boldsymbol{M}\left(\boldsymbol{v}_{n+1}-\boldsymbol{v}_{n}\right)=-\bar{\nabla} V\left(\boldsymbol{q}_{n}, \boldsymbol{q}_{n+1}\right)\left(\boldsymbol{q}_{n+1}-\boldsymbol{q}_{n}\right) \\
-\left(\boldsymbol{q}_{n+1}-\boldsymbol{q}_{n}\right) \cdot \sum_{S \in \bar{\omega}}\left(D_{1} \boldsymbol{\pi}\left(\overline{\boldsymbol{q}}_{n+\frac{1}{2}}^{S}, \boldsymbol{d}_{n+\frac{1}{2}}^{S}, \boldsymbol{f}_{n+\frac{1}{2}}^{S}\right)\right)^{T} \\
\overline{\bar{\nabla}}_{\pi} \tilde{\boldsymbol{g}}_{\left(\boldsymbol{\pi}_{n}, \boldsymbol{\pi}_{n+1}\right) \cdot \boldsymbol{\lambda}_{n+1}}
\end{gathered}
$$

The last equation can be recast in the form

$$
\begin{aligned}
& \frac{1}{2} \boldsymbol{v}_{n+1} \cdot \boldsymbol{M} \boldsymbol{v}_{n+1}-\frac{1}{2} \boldsymbol{v}_{n} \cdot \boldsymbol{M} \boldsymbol{v}_{n}=-\left[V\left(\boldsymbol{q}_{n+1}\right)-V\left(\boldsymbol{q}_{n}\right)\right] \\
& \quad+\boldsymbol{\lambda}_{n+1} \cdot \sum_{S \in \bar{\omega}}\left(D_{1} \boldsymbol{\pi}\left(\overline{\boldsymbol{q}}_{n+\frac{1}{2}}^{S}, \boldsymbol{d}_{n+\frac{1}{2}}^{S}, \boldsymbol{f}_{n+\frac{1}{2}}^{S}\right)\right)^{T} \\
& \overline{\bar{\nabla}}_{\pi} \tilde{\boldsymbol{g}}^{S}\left(\boldsymbol{\pi}_{n}, \boldsymbol{\pi}_{n+1}\right) \cdot\left(\boldsymbol{q}_{n+1}-\boldsymbol{q}_{n}\right)
\end{aligned}
$$

where the directionality property of the discrete derivative (see [10]) has been employed. To show that the last term in (66) equals zero, we have to consider the consistency condition (56) in the discrete setting, i.e. to prove that

$$
\begin{aligned}
& {\left[\left(\overline{\boldsymbol{q}}_{I, n+1}^{S}-\overline{\boldsymbol{q}}_{I, n}^{S}\right) \cdot\left(D_{1} \boldsymbol{\pi}\left(\overline{\boldsymbol{q}}_{, n+\frac{1}{2}}^{S}, \boldsymbol{d}_{n+\frac{1}{2}}^{S}, \boldsymbol{f}_{n+\frac{1}{2}}^{S}\right)\right)^{T}\right.} \\
& \quad+\left(\boldsymbol{d}_{n+1}^{S}-\boldsymbol{d}_{n}^{S}\right) \cdot\left(D_{2} \boldsymbol{\pi}\left(\overline{\boldsymbol{q}}_{n+\frac{1}{2}}^{S}, \boldsymbol{d}_{n+\frac{1}{2}}^{S}, \boldsymbol{f}_{n+\frac{1}{2}}^{S}\right)\right)^{T} \\
& \left.\quad+\left(\boldsymbol{f}_{n+1}^{S}-\boldsymbol{f}_{n}^{S}\right) \cdot\left(D_{3} \boldsymbol{\pi}\left(\overline{\boldsymbol{q}}_{n+\frac{1}{2}}^{S}, \boldsymbol{d}_{n+\frac{1}{2}}^{S}, \boldsymbol{f}_{n+\frac{1}{2}}^{S}\right)\right)^{T}\right] \\
& \left.\quad \cdot \overline{\bar{\nabla}}_{\pi} \tilde{\boldsymbol{g}}^{S} \boldsymbol{\pi}_{n}, \boldsymbol{\pi}_{n+1}\right)=0
\end{aligned}
$$

holds. Due to the specific structure of the invariants (cf. (45)), we can recast (67) as follows 
$\left(\boldsymbol{\pi}_{n+1}-\boldsymbol{\pi}_{n}\right) \cdot \overline{\bar{\nabla}}_{\pi} \tilde{\boldsymbol{g}}^{S}\left(\boldsymbol{\pi}_{n}, \boldsymbol{\pi}_{n+1}\right)=0$.

In consequence of the discrete gradient (60) the last equation can be written as

$\tilde{\boldsymbol{g}}_{n+1}^{S}-\tilde{\boldsymbol{g}}_{n}^{S}=0$.

Insertion into (66) yields

$$
\begin{aligned}
E_{n+1} & -E_{n} \\
= & -\sum_{S \in \bar{\omega}} \lambda_{S, n+1} \cdot\left(D_{2} \boldsymbol{\pi}\left(\overline{\boldsymbol{q}}_{n+\frac{1}{2}}^{S}, \boldsymbol{d}_{n+\frac{1}{2}}^{S}, \boldsymbol{f}_{n+\frac{1}{2}}^{S}\right)\right)^{T} \overline{\bar{\nabla}}_{\pi} \tilde{\boldsymbol{g}}^{S}\left(\boldsymbol{\pi}_{n}, \boldsymbol{\pi}_{n+1}\right) \\
& \left.\quad \cdot\left(\boldsymbol{d}_{n+1}^{S}-\boldsymbol{d}_{n}^{S}\right)+D_{3} \boldsymbol{\pi}\left(\overline{\boldsymbol{q}}_{n+\frac{1}{2}}^{S}, \boldsymbol{d}_{n+\frac{1}{2}}^{S}, \boldsymbol{f}_{n+\frac{1}{2}}^{S}\right)\right)^{T} \\
& \left.\quad \overline{\bar{\nabla}}_{\tilde{\pi}} \tilde{\boldsymbol{g}}^{S}\left(\boldsymbol{\pi}_{n}, \boldsymbol{\pi}_{n+1}\right) \cdot\left(\boldsymbol{f}_{n+1}^{S}-\boldsymbol{f}_{n}^{S}\right)\right) \\
= & 0
\end{aligned}
$$

where $(59)_{3}$ and $(59)_{4}$ have been taken into account. Accordingly, algorithmic conservation of energy is facilitated if the discrete persistency condition $\lambda_{S} \cdot\left(\tilde{\boldsymbol{g}}_{n+1}^{S}-\tilde{\boldsymbol{g}}_{n}^{S}\right)=0$ is fulfilled.

The active set strategy (see [18] and the references therein for details) separates the set of constraints into the active and inactive set within each time step. A constraint, moving from the inactive into the active set violates the constraint requirements at time $t_{n}$ in general. This also violates the consistency condition and (69), respectively. Different possibilities are given to deal with the problem at hand (cf. [16]). First of all, energy-momentum schemes have been developed to overcome numerical instabilities in nonlinear elastodynamics (see [28]). This requirement is fulfilled by the proposed approach independent of the violation of the consistency condition, so there is no need to correct this kind of artificial energy. If necessary (e.g. for the numerical verification of energy conservation), the consistency condition itself can be used by replacing the original constraint function according to

$\tilde{\boldsymbol{g}}_{n+1}^{S} \rightarrow \tilde{\boldsymbol{g}}_{n+1}^{S}-\tilde{\boldsymbol{g}}_{n}^{S}$

so that condition (69) is always satisfied.

\section{Numerical investigations}

In this section we investigate the numerical performance of the newly developed energy-momentum scheme. A model problem is used for the demonstration of the basic concepts. Then two impact simulations are presented.

\subsection{Nonlinear spring}

As a first example we use this simple constrained non-contact model problem to demonstrate the properties of the time integration scheme. Generally, the large deformation inequality

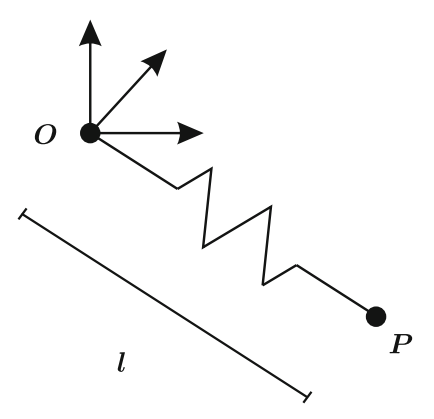

Fig. 3 Nonlinear spring

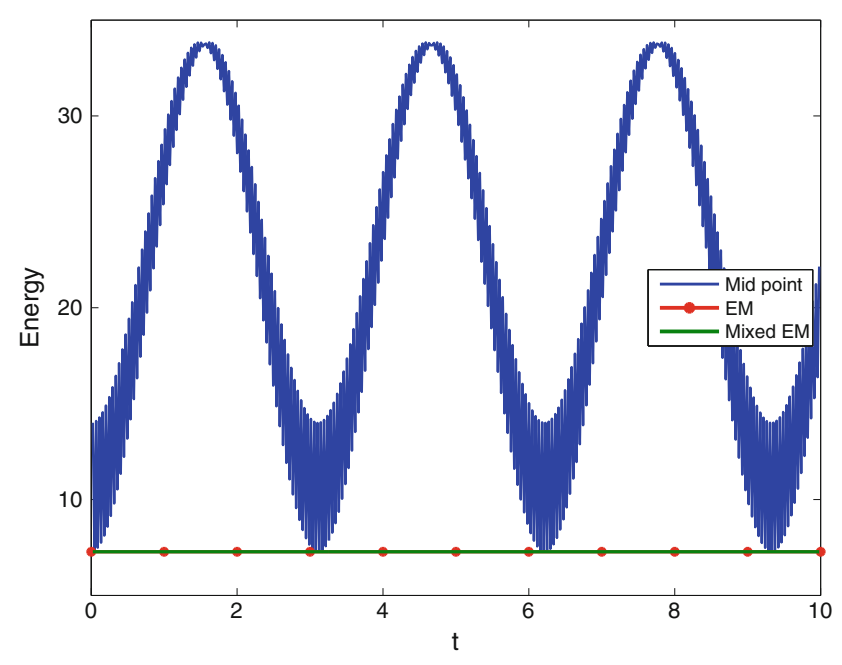

Fig. 4 Total energy versus time. Note, that the red curve is congruent to the green line

constraints possess a similar behavior in time, once a specific constraint is active.

The three-dimensional model problem consists of a point mass $\boldsymbol{P}$ with coordinates $\boldsymbol{q}=[x, y, z]$ and unit mass $m=1$ (see Fig. 3). The point mass is connected to the point of origin $\boldsymbol{O}$ by a nonlinear spring, using a spring-potential $V(\nu)=$ $\frac{1}{8}\left(v-\frac{1}{v}\right) c\left(v-\frac{1}{v}\right)$, where $c=10^{6}$ is constant, and $v=\boldsymbol{q} \cdot \boldsymbol{q} / l^{2}$ denotes a measure of stretch and hence a proper invariant. Here, $l=\|\boldsymbol{q}\|_{t=0}$ is the reference length of the spring. The initial values are $\boldsymbol{q}=[0.5,0.5,0.7]$ and $\dot{\boldsymbol{q}}=[1.5,3,-2] \times \boldsymbol{q}$. Furthermore, the chosen time-step size $\Delta t=0.02263 \mathrm{spec}-$ ifies the largest possible value if a mid-point evaluation is used. The Lagrangian reads

$L(\boldsymbol{q}, \dot{\boldsymbol{q}})=T-V$

with the kinetic energy $T=\frac{1}{2} \dot{\boldsymbol{q}} \cdot \boldsymbol{M} \dot{\boldsymbol{q}}, \boldsymbol{M}=m \boldsymbol{I}$ and $\boldsymbol{I} \in$ $\mathbb{R}^{n \times n}$ is the $n$-dimensional unity matrix.

In the following, we demonstrate the advantages of an energy-momentum scheme in comparison with a mid-point integration using this model problem. Furthermore, we demonstrate how to rewrite the system using augmented coordinates, i.e. a mixed approach based on a $\mathrm{Hu}$-Washizu potential 

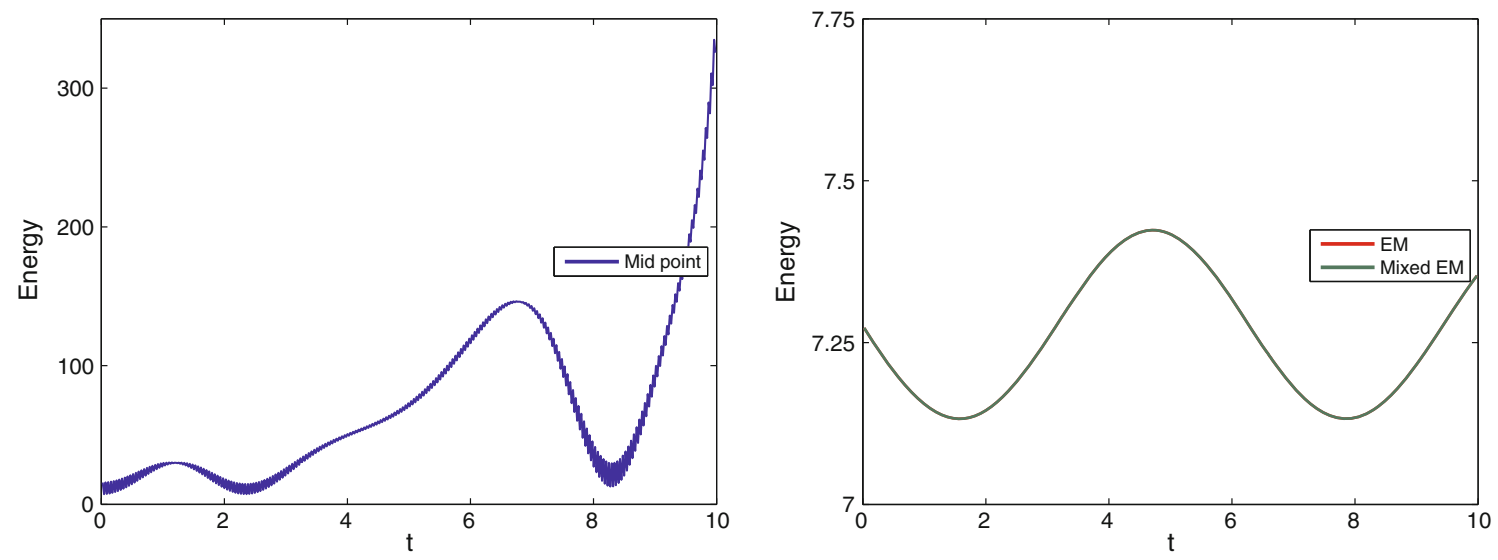

Fig. 5 Total energy versus time

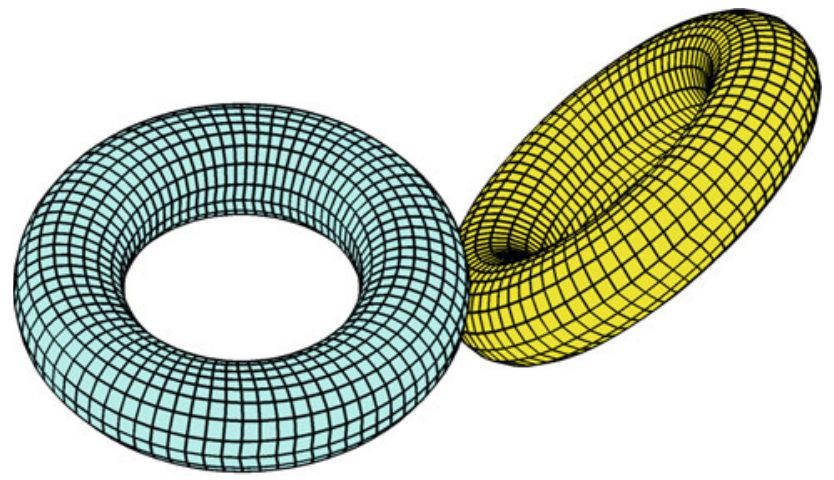

based on the invariant $\pi=v$ and

$\overline{\bar{\nabla}}_{\pi} V\left(\pi_{n}, \pi_{n+1}\right)=\frac{V\left(\pi_{n+1}\right)-V\left(\pi_{n}\right)}{\pi_{n+1}-\pi_{n}}$.

As can be seen in Fig. 4 (EM), energy is conserved exactly.

Mixed approach Next, we formulate a mixed approach by introducing the strain measure $v$ as an additional augmented variable to the system. In particular, this formulation equals a $\mathrm{Hu}$-Washizu type formulation of the system. For the Lagrangian follows

$L(\boldsymbol{q}, \dot{\boldsymbol{q}}, v, \lambda)=T-(V(v)+\lambda \Phi)$

Fig. 6 Initial configuration of the two tori impact problem

and the advantages thereof. In a last step, we simulate the inconsistency mentioned in Sect. 5.1 by disturbing the constraints of the mixed approach.

Mid-Point rule The equation of motion are given by

$\mathbf{0}=\frac{\mathrm{d}}{\mathrm{d} t}\left(\frac{\mathrm{d} L}{\dot{\boldsymbol{q}}}\right)-\frac{\mathrm{d} L}{\boldsymbol{q}}$.

Thus, the implementation of a mid-point evaluation reads

$$
\begin{aligned}
\mathbf{0}= & \frac{2}{\Delta t} \boldsymbol{M}\left(\boldsymbol{q}_{n+1}-\boldsymbol{q}_{n}-\Delta t \boldsymbol{v}_{n}\right) \\
& +\frac{\Delta t}{2} c\left(v_{n+1 / 2}-\frac{1}{v_{n+1 / 2}^{3}}\right) \frac{\boldsymbol{q}_{n+1 / 2}}{l^{2}}
\end{aligned}
$$

with $v_{n+1 / 2}=\frac{\boldsymbol{q}_{n+1 / 2} \cdot \boldsymbol{q}_{n+1 / 2}}{l^{2}}$ and $\boldsymbol{q}_{n+1 / 2}=\frac{1}{2}\left(\boldsymbol{q}_{n+1}+\boldsymbol{q}_{n}\right)$. As can be seen in Fig. 4 (Mid point), we obtain large oscillations in the total energy.

Energy-momentum scheme The advocated approach to improve the performance of the time integration scheme is based on the concept of discrete gradient, as proposed in Gonzalez [10]. Consequently, (74) is replaced with

$\mathbf{0}=\frac{2}{\Delta t} \boldsymbol{M}\left(\boldsymbol{q}_{n+1}-\boldsymbol{q}_{n}-\Delta t \boldsymbol{v}_{n}\right)+\Delta t D_{1} \pi(\boldsymbol{q})^{T} \overline{\bar{\nabla}}_{\pi} V\left(\pi_{n}, \pi_{n+1}\right)$

$\Phi=v-\frac{\boldsymbol{q} \cdot \boldsymbol{q}}{l^{2}}$

The equations of motion can be written as follows

$\mathbf{0}=\left[\begin{array}{c}\frac{2}{\Delta t} \boldsymbol{M}\left(\boldsymbol{q}_{n+1}-\boldsymbol{q}_{n}-\Delta t \boldsymbol{v}_{n}\right)+\Delta t \lambda_{n, n+1} 2 \frac{\boldsymbol{q}_{n+1 / 2}}{l^{2}} \\ \overline{\overline{\nabla_{v}}} V\left(v_{n}, v_{n+1}\right)-\lambda_{n, n+1} \\ v_{n+1}-\boldsymbol{q}_{n+1} \cdot \boldsymbol{q}_{n+1} / l^{2}\end{array}\right]$

using a mid-point evaluation of the configuration $\boldsymbol{q}_{n+1 / 2}=$ $\frac{1}{2}\left(\boldsymbol{q}_{n+1}+\boldsymbol{q}_{n}\right)$ and a constant Lagrangian multiplier $\lambda_{n, n+1}$. The discrete gradient can be defined as in (76). Indeed, the mixed formulation (79) can be easily reduced to the EM scheme (75). Note, however, that the introduction of additional augmented variables leads to a structural simplification of the resulting equation in (79).

To simulate the behavior of the inconsistency of the constraints caused by the discrete active set strategy, we induce a sinusoidal disturbance $l^{\star}=l+0.01 \sin (t)$ into the reference length of the spring. Thereupon the constraint $(79)_{3}$ will not be satisfied in any time-step at time $t_{n}$, which is structurally the same type of inconsistency resulting from the active set strategy. The left diagram in Fig. 5 shows the result of the mid-point evaluation. What we receive is a blow up in 

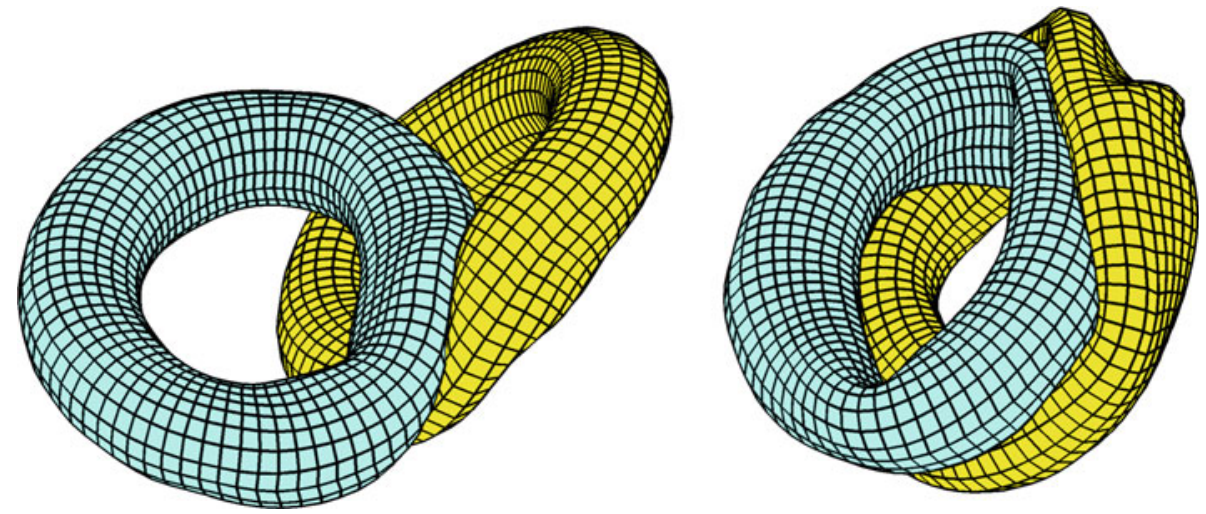

Fig. 7 Deformation at time $t=2.5$ and $t=5$

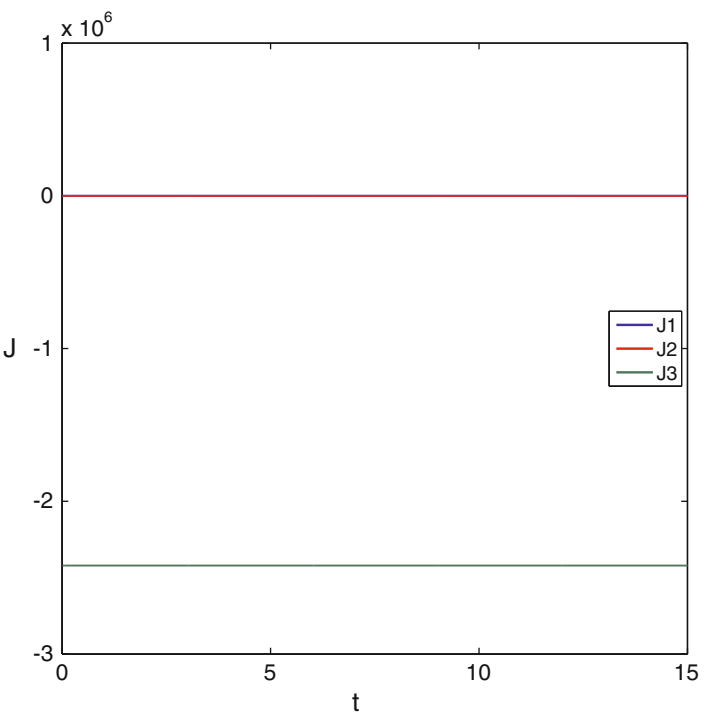

Fig. 8 Total linear, angular momentum and total energy

total energy. As expected, the energy-momentum schemes remain stable as shown in the lower right diagram in Fig. 5. Furthermore, the results of both energy-momentum schemes are congruent up to some numerical fluctuations.

\subsection{Two tori impact problem}

In this example we consider an impact problem of two tori. Initial values and the material properties have been taken from Yang and Laursen [33]. The initial configuration is displayed in Fig. 6, the inner and outer radius of the tori are 52 and 100, the wall thickness of each hollow torus is 4.5. Both tori are subdivided into 3,120 elements, using a NeoHookean hyperelastic material with $\mathcal{E}=2,250$ and $v=0.3$. The initial densities are $\rho=0.1$ and the homogeneous, initial velocity of the left torus is given by $\boldsymbol{v}=[30,0,23]$.

A time-step size of $\Delta t=0.01$ has been used to calculate 1,500 time steps. A sequence of deformed configurations at $t=2.5$ and $t=5$ is displayed in Fig. 7. Since no external forces are acting, the system under consideration
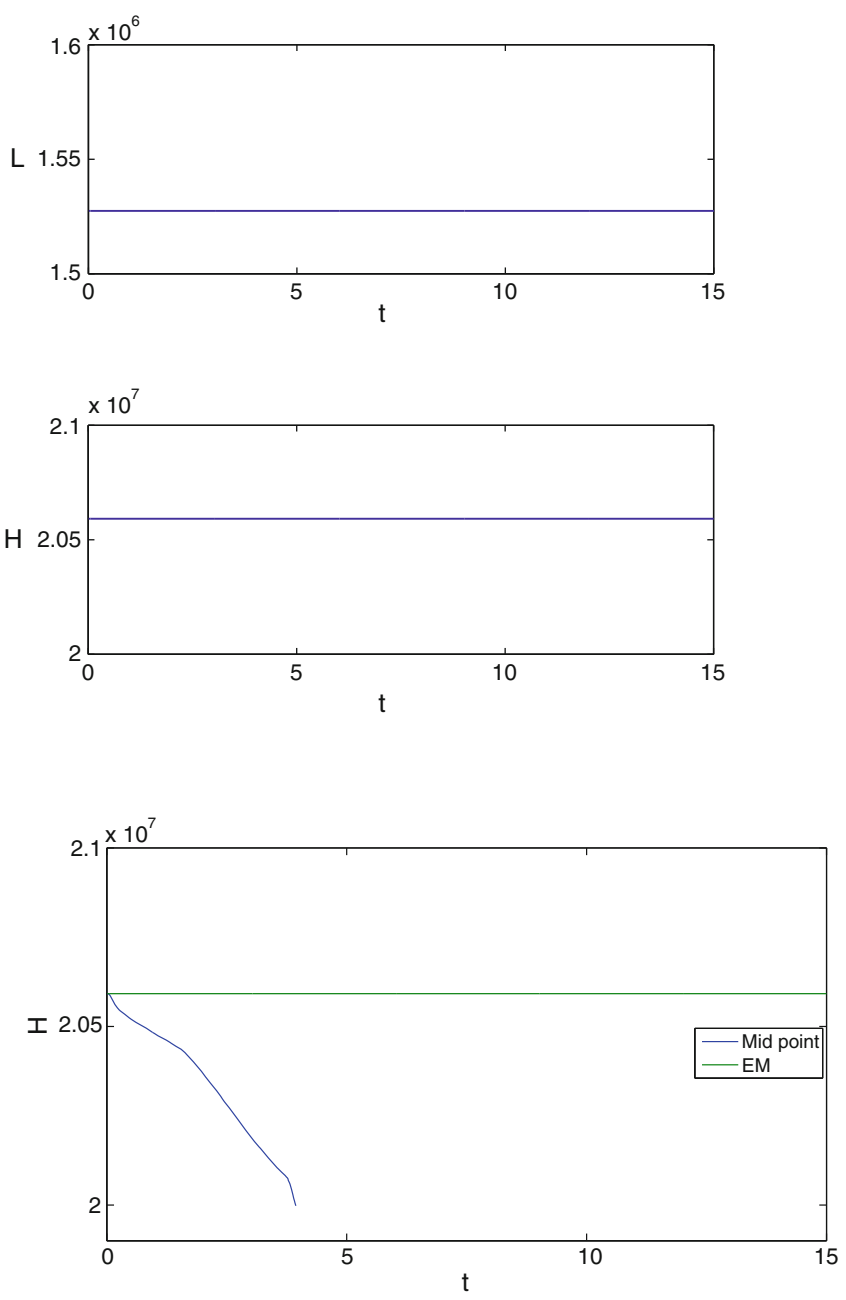

Fig. 9 Total linear, angular momentum and total energy

can be classified as a Hamiltonian system with symmetries. The evolution of the total angular momentum is depicted in the left diagram of Fig. 8. All three components $\left[J_{1}, J_{2}, J_{3}\right]$ are displayed. Due to the initial values, the third component $\left(J_{3} \approx-2.4 \times 10^{6}\right)$ is larger than the other two components $\left(J_{1} \approx 2.15 \times 10^{2}\right)$ and $\left(J_{1} \approx-2.15 \times 10^{2}\right)$. In addition to 

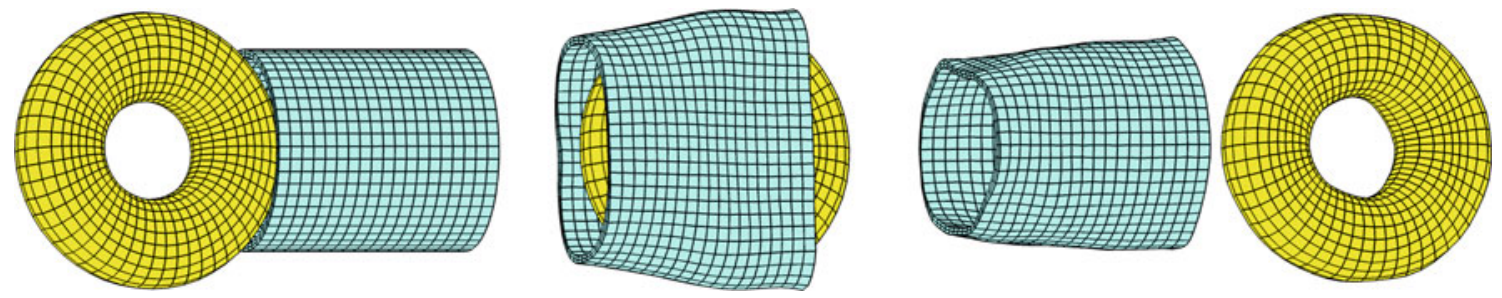

Fig. 10 Deformed configuration at $t=[0,20,50]$
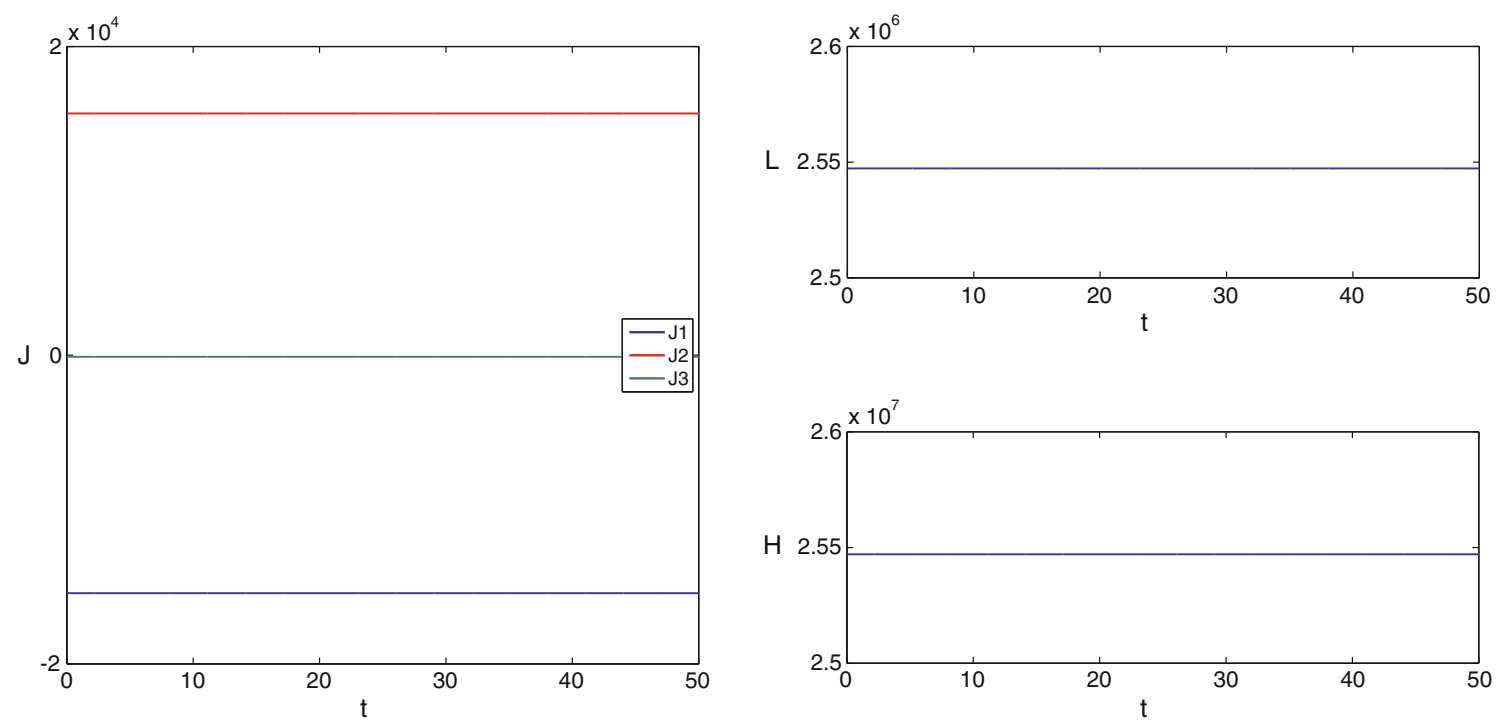

Fig. 11 Total linear, angular momentum and total energy

this, the upper and lower right diagrams show the total linear momentum and the total energy, respectively. As expected, all quantities are conserved up to numerical round-off.

Note, that we have used the modification described in (71) for this simulation. Without this modification, linear and angular momentum are conserved as well, only total energy shows some minor artificial changes. The simulation itself remains stable.

In contrast, the standard mid-point scheme fails to conserve energy. Figure 9 shows a comparison of the proposed energy-momentum scheme and the mid-point type rule. As can be seen, the newton iteration diverges after 394 time steps.

Note that the characteristic of the energy-curve of the midpoint type approach depends highly on the implementation of the active set strategy.

\subsection{Torus-cylinder impact example}

This last example deals with a three-dimensional problem similar to the last example in Yang and Laursen [33]. The material properties and the initial geometry of the torus are the same as in Sect. 6.2. The inner diameter of the cylinder is 100 , the wall thickness is 7.5 and the initial velocity of the torus is $[0,0,20]$. In Fig. 10 a sequence of configurations at $t=0,20,50$ is displayed. The torus consists of 2,288 elements, the cylinder of 1,900 elements. Furthermore, the time-step size has been set to $\Delta t=0.01$.

Once again, the evolution of the total angular momentum is depicted in the left diagram of Fig, 11, whereas the upper and lower diagrams on the right side show the total linear momentum and the total energy, respectively. As expected, all quantities are conserved exactly.

\section{Conclusions}

We have presented a newly developed energy-momentum scheme for the NTS method in the context of large deformations. In particular, the NTS constraints have been reformulated using a mixed approach in conjunction with the concept of a discrete gradient. This ensures algorithmic conservation of energy as well as both momentum maps, leading to an enhanced numerical stability while preserving the second order accuracy of the original mid-point type time integration. The enhanced numerical stability is guaranteed even if the non-penetration condition is enforced exactly. Due to the simple structure of the proposed algorithm, the presented 
concept is well-suited for the extension to frictional contact problems. Furthermore, due to the newly proposed augmentation technique, the first and second derivative of the convective coordinates required for detecting the contact points are-in contrast to traditional methods-quite simple.

Several numerical examples are presented to demonstrate the characteristics of the proposed algorithm. In particular, the numerical results confirm the enhanced numerical stability and robustness of the newly developed method.

Acknowledgments Support for this research was provided by the Deutsche Forschungsgemeinschaft (DFG) under grant HE 5943/1-1. This support is gratefully acknowledged.

\section{References}

1. Armero F, Petöcz E (1998) Formulation and analysis of conserving algorithms for frictionless dynamic contact/impact problems. Comput Methods Appl Mech Eng 158:269-300

2. Armero F, Romero I (2001) On the formulation of high-frequency dissipative time-stepping algorithms for nonlinear dynamics. Part I: low-order methods for two model problems and nonlinear elastodynamics. Comput Methods Appl Mech Eng 190:2603-2649

3. Armero F, Romero I (2001) On the formulation of high-frequency dissipative time-stepping algorithms for nonlinear dynamics. Part II: second-order methods. Comput Methods Appl Mech Eng 190:6783-6824

4. Benson DJ, Hallquist JO (1990) A single surface contact algorithm for the post-buckling analysis of shell structures. Comput Methods Appl Mech Eng 78:141-163

5. Betsch P, Hesch C (2007) Energy-momentum conserving schemes for frictionless dynamic contact problems. Part I: NTS method. In: Wriggers P, Nackenhorst U (eds) IUTAM symposium on computational methods in contact mechanics, vol 3 of IUTAM Bookseries. Springer, Berlin, pp 77-96

6. Betsch P, Steinmann P (2000) Conserving properties of a time FE method-part I: time-stepping schemes for n-body problems. Int J Numer Methods Eng 49:599-638

7. Betsch P, Steinmann P (2001) Conserving properties of a time FE method-part II: time-stepping schemes for non-linear elastodynamics. Int J Numer Methods Eng 50:1931-1955

8. Betsch P, Steinmann P (2002) Conservation properties of a time FE method. Part III: mechanical systems with holonomic constraints. Int J Numer Methods Eng 53:2271-2304

9. Betsch P, Uhlar S (2007) Energy-momentum conserving integration of multibody dynamics. Multibody Syst Dyn 17(4):243-289

10. Gonzalez O (1996) Time integration and discrete Hamiltonian systems. J Nonlinear Sci 6:449-467

11. Gonzalez O (1999) Mechanical systems subject to holonomic constraints: Differential - algebraic formulations and conservative integration. Physica D 132:165-174

12. Gonzalez O (2000) Exact energy and momentum conserving algorithms for general models in nonlinear elasticity. Comput Methods Appl Mech Eng 190:1763-1783

13. Haikal G, Hjelmstad KD (2007) A finite element formulation of non-smooth contact based on oriented volumes for quadrilateral and hexahedral elements. Comput Methods Appl Mech Eng 196:4690-4711

14. Hallquist JO (1979) Nike2d. Technical Report UCRL-52678. University of California, Lawrence Livermore National Laboratory
15. Hauret $\mathrm{P}$, LeTallec $\mathrm{P}$ (2006) Energy-controlling time integration methods for nonlinear elastodynamics and low-velocity impact. Comput Methods Appl Mech Eng 195:4890-4916

16. Hesch C, Betsch P (2009) A mortar method for energy-momentum conserving schemes in frictionless dynamic contact problems. Int J Numer Methods Eng 77:1468-1500

17. Hesch C, Betsch P (2010) Transient 3d domain decomposition problems: frame-indifferent mortar constraints and conserving integration. Int J Numer Methods Eng 82:329-358

18. Hüeber S, Wohlmuth BI (2005) A primal-dual active set strategy for non-linear multibody contact problems. Comput Methods Appl Mech Eng 194:3147-3166

19. Hüeber S, Wohlmuth BI (2006) Mortar methods for contact problems. In: Wriggers P, Nackenhorst U (eds) Analysis and simulation of contact problems. Lecture notes in applied and computational mechanics. vol 27. Springer, Berlin, pp 39-47

20. Hughes TJR (2000) The finite element method. Dover Publications, New York

21. Konyukhov A, Schweizerhof K (2005) Covariant description for frictional contact problems. Comput Methods Appl Mech Eng 35:190-213

22. Konyukhov A, Schweizerhof K (2006) Covariant description of contact interfaces considering anisotropy for adhesion and friction: Part 1. Formulation and analysis of the computational model. Comput Methods Appl Mech Eng 196:103-117

23. Laursen TA (1992) Formulation and treatment of frictional contact problems using finite elements, $\mathrm{PhD}$ thesis. Stanford University

24. Laursen TA (2002) Computational contact and impact mechanics. Springer, Berlin

25. Laursen TA, Chawla V (1997) Design of energy conserving algorithms for frictionless dynamic contact problems. Int $\mathrm{J}$ Numer Methods Eng 40:863-886

26. Laursen TA, Love GR (2002) Improved implicit integrators for transient impact problems - geometric admissibility within the conserving framework. Int J Numer Methods Eng 53:245-274

27. Puso MA, Laursen TA, Solberg JM (2008) A segment-to-segment mortar contact method for quadratic elements and large deformations. Comput Methods Appl Mech Eng 197:555-566

28. Simo JC, Tarnow N (1992) The discrete energy-momentum method. Conserving algorithms for nonlinear elastodynamics. Z Angew Math Phys 43:757-792

29. Simo JC, Tarnow N, Wong KK (1992) Exact energy-momentum conserving algorithms and symplectic schemes for nonlinear dynamics. Comput Methods Appl Mech Eng 100:63-116

30. Tur M, Fuenmayor FJ, Wriggers P (2009) A mortar-based frictional contact formulation for large deformations using Lagrange multipliers. Comput Methods Appl Mech Eng 198:2860-2873

31. Wriggers $P$ (2006) Computational contact mechanics, 2nd edn. Springer, Berlin

32. Wriggers P, Van TV, Stein E (1990) Finite element formulations of large deformation impact-contact problems with friction. Comput Struct 37:319-331

33. Yang B, Laursen TA (2008) A contact searching algorithm including bounding volume trees applied to finite sliding mortar formulation. Comput Mech 41:189-205

34. Yang B, Laursen TA (2008) A large deformation mortar formulation of self contact with finite sliding. Comput Methods Appl Mech Eng 197:756-772

35. Zienkiewicz OC, Taylor RL (2005) The finite element method for solid and structural mechanics, 6th edn. Butterworth Heinemann, London

36. Zienkiewicz OC, Taylor RL, Zhu JZ (2005) The finite element method. Its basis and fundamentals, 6th edn. Butterworth Heinemann, London 